ARTICLE

\title{
ANGPTL4 deficiency in haematopoietic cells promotes monocyte expansion and atherosclerosis progression
}

Binod Aryal ${ }^{1,2,3}$, Noemi Rotllan ${ }^{1,2, \star}$, Elisa Araldi ${ }^{1,2,}$, Cristina M. Ramírez ${ }^{1,2}$, Shun He ${ }^{4}$, Benjamin G. Chousterman ${ }^{4}$, Ashley M. Fenn ${ }^{4}$, Amarylis Wanschel ${ }^{3}$, Julio Madrigal-Matute ${ }^{3}$, Nikhil Warrier ${ }^{3}$, Jose L. Martín-Ventura ${ }^{5}$, Filip K. Swirski ${ }^{4}$, Yajaira Suárez ${ }^{1,2} \&$ Carlos Fernández-Hernando ${ }^{1,2}$

Lipid accumulation in macrophages has profound effects on macrophage gene expression and contributes to the development of atherosclerosis. Here, we report that angiopoietin-like protein 4 (ANGPTL4) is the most highly upregulated gene in foamy macrophages and it's absence in haematopoietic cells results in larger atherosclerotic plaques, characterized by bigger necrotic core areas and increased macrophage apoptosis. Furthermore, hyperlipidemic mice deficient in haematopoietic ANGPTL4 have higher blood leukocyte counts, which is associated with an increase in the common myeloid progenitor (CMP) population. ANGPTL4-deficient CMPs have higher lipid raft content, are more proliferative and less apoptotic compared with the wild-type (WT) CMPs. Finally, we observe that ANGPTL4 deficiency in macrophages promotes foam cell formation by enhancing CD36 expression and reducing $A B C A 1$ localization in the cell surface. Altogether, these findings demonstrate that haematopoietic ANGPTL4 deficiency increases atherogenesis through regulating myeloid progenitor cell expansion and differentiation, foam cell formation and vascular inflammation.

\footnotetext{
${ }^{1}$ Vascular Biology and Therapeutics Program, Yale University School of Medicine, New Haven, Connecticut 06520, USA. ${ }^{2}$ Integrative Cell Signaling and Neurobiology of Metabolism Program, Section of Comparative Medicine and Department of Pathology, Yale University School of Medicine, New Haven, Connecticut 06520, USA. ${ }^{3}$ Departments of Medicine and Cell Biology, Leon H. Charney Division of Cardiology and Cell Biology, New York University School of Medicine, New York, New York 10016, USA. ${ }^{4}$ Center for Systems Biology, Massachusetts General Hospital and Harvard Medical School, Boston, Massachusetts 02114, USA. ${ }^{5}$ Vascular Research Lab, IIS-Fundación Jimenez-Díaz, Universidad Autónoma de Madrid, Madrid 28040, Spain. * These authors contributed equally to this work. Correspondence and requests for materials should be addressed to C. F.-H. (email: carlos.fernandez@yale.edu) or to Y.S (yajaira.suarez@yale.edu).
} 
$\mathrm{D}$ uring the early stages of atherosclerosis, modified lipoproteins, primarily oxidized low density lipoproteins (Ox-LDL) accumulate in the intima, and activate endothelial and smooth muscle cells, recruit circulating monocytes into the sub-endothelial layer. Here, monocytes differentiate into macrophages, scavenge Ox-LDL, accumulate neutral lipids and transform into foam cells ${ }^{1,2}$. Foam cell formation is a protective mechanism whereby the vessel wall rids itself of potentially harmful lipids. However, accumulation of large numbers of foam cells in the arterial wall leads to the generation of atherosclerotic plaques ${ }^{1}$. Furthermore, both macrophages and foam cells play a key role in mediating inflammatory response in athero-plaques. Apart from foam cells, the monocyte count in blood circulation independently predicts risk for coronary artery disease after adjustment for conventional risk factors ${ }^{3}$. Monocytosis and neutrophilia have been observed in animal models of atherosclerosis including pigs and rabbits, and seem to contribute to atherogenesis ${ }^{4,5}$. Previous studies have demonstrated that hyperlipidemia-induced leukocytosis in different mouse models including $A b c a 1^{-/-}$or $A b c g 1^{-/-}$ and $A \mathrm{poe}^{-1-}$ mice is associated with the expansion and proliferation of haematopoietic stem and multipotential progenitor cells (HSPCs) in the bone marrow $(\mathrm{BM})^{6-8}$. Recent studies have shown that a family of proteins called angiopoietinlike proteins (ANGPTLs), particularly ANGPTL2 and ANGPTL5, are known to stimulate the expansion of haematopoietic stem cells ex $v i v o^{9-11}$. In addition, ANGPTL4 has been shown maintains in vivo repopulation capacity of $\mathrm{CD} 34+$ human cord blood cells ${ }^{12}$.

ANGPTL4 is a multifunctional protein that regulates many metabolic and non-metabolic processes through its distinct $\mathrm{N}$-terminal and C-terminal domains ${ }^{13-17}$. Particularly, ANGPTL4 is a strong inhibitor of lipoprotein lipase (LPL), an enzyme that catalyses the hydrolysis of triglycerides (TG) from very LDL (VLDL) and chylomicrons, and regulates the uptake of circulating lipids into tissues ${ }^{18,19}$. As a result, overexpression of ANGPTL4 in mice leads to hypertriglyceridemia, whereas deficiency leads to lowering of circulating lipids ${ }^{20}$. Interestingly, human studies have shown that a common sequence variant near the ANGPTL4 gene is associated with decreased plasma TGs and increased high-density lipoprotein cholesterol (HDL-C) levels, and ANGPTL4 expression is positively associated with metabolic parameters including levels of insulin, fatty acids and leptin ${ }^{21}$. Although decreased lipid content is generally atheroprotective, E40K, a loss of function variant of ANGPTL4, was associated with increased coronary heart disease risk despite being associated with an atheroprotective lipid profile, suggesting that ANGPTL4 influences parameters beyond lipid levels ${ }^{22}$.

ANGPTL4 is highly expressed in adipose tissues, placenta, liver, kidney and heart in humans $s^{23,24}$. It's expression is regulated by fasting, PPARs, glucocorticoids, and hypoxia in a tissuespecific manner $23-26$. ANGPTL4 is also highly abundant in macrophages and it is induced by dietary fatty acids ${ }^{27}$. Angptl4 ${ }^{-1-}$ mice develop severe inflammation and accumulate foam cells in the mesenteric lymph nodes when fed a diet high in saturated fat ${ }^{27}$. This suggests that ANGPTL4 is a critical regulator of macrophage functions. Moreover, studies from overexpression or depletion of LPL in macrophages demonstrate that LPL promotes the binding and uptake of modified LDLs by macrophages and thus enhances foam cell formation ${ }^{28,29}$. ANGPTL4 can be expected to inhibit and reverse LPL-mediated effects in macrophages and atherosclerosis. However, there have been no studies addressing the direct role of macrophage ANGPTL4 during atherogenesis. Studies using global knockout or transgenic overexpression mouse models suggest both pro- and anti-atherogenic roles of ANGPTL4 (refs 30,31). These confounding observations could have resulted from diverse roles of ANGPTL4 in regulating multiple metabolic parameters and inflammation, which could influence the progression of atherosclerosis.

In the present study, we demonstrate that haematopoieticspecific ANGPTL4 plays a critical role in the progression of atherosclerosis. We show that haematopoietic ANGPTL4 deficiency in $L d l r^{-/-}$mice results in accelerated atherosclerosis characterized by bigger lesions, enhanced lipid accumulation, vascular inflammation and increased leukocytes in circulation. In doing so, we uncover a novel role of ANGPTL4 in the regulation of common myeloid progenitor (CMP) expansion and its subsequent differentiation into monocytes and neutrophils. We also provide additional mechanisms showing that ANGPTL4mediated suppression of foam cell formation is a multifactorial process, involving increased lipoprotein influx and decreased cholesterol efflux from macrophages.

\section{Results}

ANGPTL 4 is expressed in macrophages in atherosclerotic plaques. We initially aimed to identify genes that are modulated in macrophage-derived foam cells. To this end, we loaded thioglycollate-elicited mouse peritoneal macrophages with acetylated LDL (Ac-LDL), a synthetically modified form of native LDL to maximize cholesterol loading. Genes regulated at the mRNA level by cholesterol loading were then determined using an Affymetrix expression array (Supplementary Table 1). In addition to genes that have previously been associated with cholesterol metabolism, including Cyp51, Dhcr24 and Sqle (Fig. 1a), we found a number of novel genes upregulated in macrophages incubated with Ac-LDL (fold change >1.4; $P<0.05$, unpaired $t$-test) (Fig. 1a). To confirm the results from the microarray study, a panel of 10 genes, that were identified as being highly upregulated upon cholesterol loading, were independently validated using quantitative real-time PCR (qRT-PCR) (Fig. 1b). Intriguingly, both microarray and qRT-PCR analysis revealed that Angptl4 was the most highly and consistently upregulated gene in macrophage-derived foam cells (Fig. 1a,b).

We next measured the expression of ANGPTL4 in peritoneal macrophages isolated from $\mathrm{Ldlr}^{-/-}$mice fed a Western-type diet (WD), which serves as an in vivo model of macrophage foam cells. As expected, macrophages isolated from $\mathrm{Ldlr}^{-/}-$mice showed a marked up-regulation of $A b c a 1$ and downregulation of Hmgcr compared with those from wild-type (WT) mice, indicating a massive accumulation of cholesterol (Fig. 1c). Importantly, Angptl4 levels were significantly upregulated in macrophages and the whole aorta of $L d l r^{-/}$mice compared with WT mice (Fig. 1c). We also confirmed these results in primary human peripheral blood mononuclear cells (PBMC) treated with Ac-LDL (Fig. 1d). Notably, immunohistochemistry analysis of mouse aortic sinus plaques (Fig. 1e) and human carotid artery plaques (Fig. 1f) revealed a significant colocalization of ANGPTL4 and CD68, a macrophage marker, confirming that ANGPTL4 is highly expressed in macrophages in atherosclerotic plaques. Moreover, culprit plaques (CPs) from human carotid arteries (characterized by an advanced plaque area with massive infiltration of macrophages) showed significantly higher expression of ANGPTL4 at both the mRNA and protein levels compared with their adjacent non-complicated plaques (NCPs) (characterized by fibrotic areas with less inflammation) (Fig. 1g,h). In addition to the full-length ANGPTL4 $(\sim 50 \mathrm{kDa})$ band, Western blot analysis showed stronger $(\sim 75 \mathrm{kDa})$ and fainter $(\sim 30 \mathrm{kD})$ bands, possibly representing oligomeric and individual truncated forms of ANGPTL4, as reported in previous studies ${ }^{19}$. Collectively, these data demonstrate that accumulation of lipids, which characterizes the formation of 
a
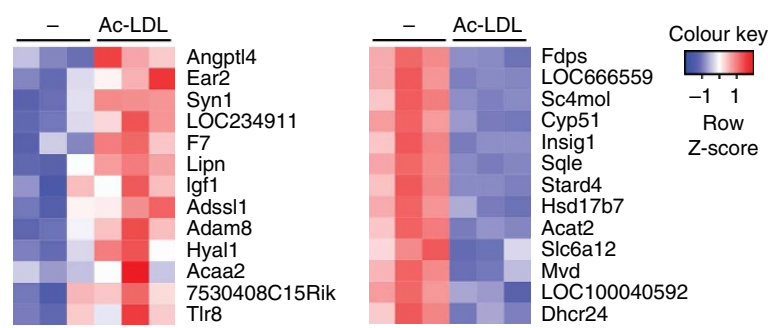

C
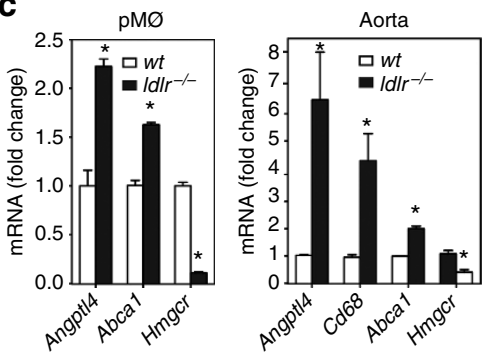

f

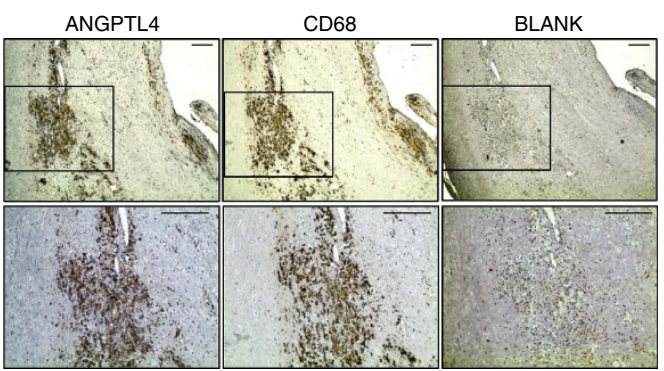

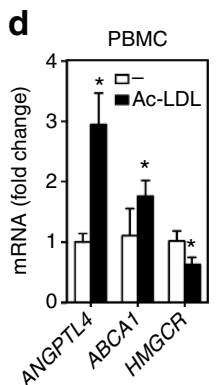

b

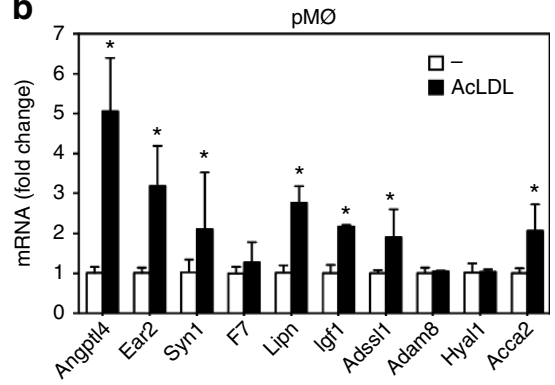

e

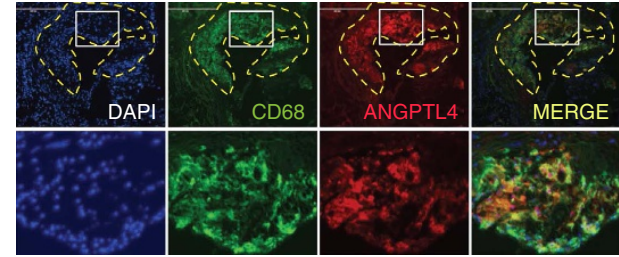

g

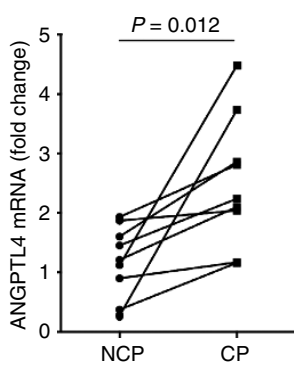

h
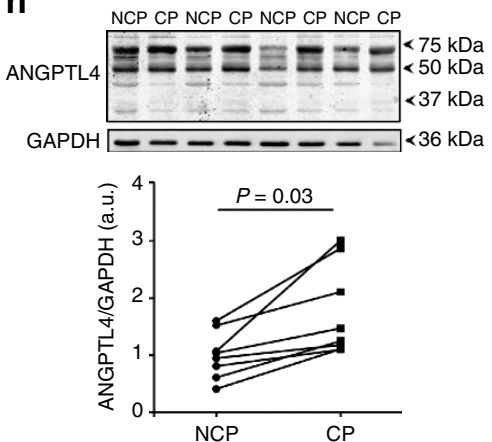

Figure 1 | ANGPTL4 is expressed in macrophages accumulated in atherosclerotic plaques. (a) Heat map representation of gene expression from microarray data comparing mouse peritoneal macrophages incubated with or without Ac-LDL $\left(120 \mu \mathrm{g} \mathrm{ml}^{-1}\right)$ for $24 \mathrm{~h}$. Left panel shows upregulated genes $(P<0.05$, unpaired $t$-test; fold change $\geq 1.4)$ and right panel shows downregulated genes $(P<0.05$, unpaired $t$-test; fold change $\geq 2.08)$ upon $A c-L D L$ treatment compared with non-treated cells. Samples were analysed in triplicate. (b) qRT-PCR validation of selected genes upregulated in the microarray in mouse peritoneal macrophages treated with or without Ac-LDL for $24 \mathrm{~h}$. qRT-PCR analysis of Angpt/4 expression levels in macrophages (c, left) and whole aorta (c, right) from WT and Ldlr ${ }^{-/}$- mice fed a WD (left) ( $n=4$ per group), and in human peripheral blood mononuclear cells (d) treated with or without Ac-LDL $\left(120 \mu \mathrm{g} \mathrm{ml}^{-1}\right)$ for $24 \mathrm{~h}$. Abcal and Hmgcr genes were used as control genes for cholesterol loading and Cd68 was used as a marker for macrophages. All data represent the mean \pm s.e.m. and are representative of three experiments in duplicate; ${ }^{\star} P \leq 0.05$ compared with untreated macrophages (b,d) and WT mice (c) by unpaired t-test. (e) Immunostaining of ANGPTL4 (red) and macrophage marker CD68 (green) and their co-localization in atherosclerotic plaques of $\mathrm{Ldll}^{-/-}$mice fed a WD. Scale bar, $400 \mu \mathrm{m}$. (f) Immunohistochemistry staining of ANGPTL4 and CD68 in human atherosclerotic plaques. Scale bar, $200 \mu \mathrm{m}$. (g) Comparison of ANGPTL4 mRNA expression in NCP and corresponding culprit (CP) human atherosclerotic plaques $(n=9)$. (h) Representative western blot showing comparison of ANGPTL4 expression in NCP and corresponding CP. Lower panel shows densitometry analysis for the $50 \mathrm{kDa}$ bands of the western blots $(n=7)$. ${ }^{\star} P \leq 0.05$ compared with NCP by unpaired $t$-test; a.u. arbitrary units. Full scans of westerns blots are provided in Supplementary Fig. 7.

macrophage-derived foam cells, also results in enhanced expression of ANGPTL4 in vitro and in vivo.

Scavenger receptors, including CD36 and SR-A, mediate modified lipoprotein uptake in macrophages ${ }^{32,33}$. To determine whether Ac-LDL regulates Angptl4 expression in a CD36dependent manner, macrophages isolated from WT and Cd $36^{-/-}$mice were treated with Ac-LDL and Angptl4 expression was compared. Surprisingly, there was no difference in the expression of Angptl4, indicating that CD36 is dispensable for this process (Supplementary Fig. 1a). Importantly, Ox-LDL, but not free cholesterol loading using cyclodextrin (Chol-CD), also induced Angptl4 expression in macrophages, suggesting that additional species, such as fatty acids within Ac-LDL or Ox-LDL rather than cholesterol, might be involved in this induction
(Supplementary Fig. 1b). Indeed, previous reports have demonstrated that members of a subfamily of the nuclear receptors, the PPARs, which are known to regulate Angptl4 expression in several cell types ${ }^{23,24,34}$, are able to sense and interpret fatty acid signals derived from dietary lipids and pathogenic lipoproteins ${ }^{35}$. Consistent with these observations, Ac-LDL enhanced human ANGPTL4 PPAR response element (PPRE) activity in Raw264.7 cells, indicating that Ac-LDL increased Angptl4 expression via PPAR signalling (Supplementary Fig. 1c).

The presence of areas of low oxygen tension (hypoxia) is a common feature of atherosclerotic lesions ${ }^{36,37}$. Angptl4 is a HIF- $1 \alpha$ target gene and is known to be elevated under hypoxic conditions in a number of cell types ${ }^{26,38,39}$. To determine whether 
hypoxia stimulates Angptl4 expression in macrophages as well, we exposed mouse peritoneal macrophages to hypoxic conditions using hypoxic chambers or $\mathrm{CoCl}_{2}$, a chemical inducer of HIF- $1 \alpha$. Angptl4 expression was upregulated under both conditions, along with HIF- $1 \alpha$ responsive genes Glut1 and Nos2 (Supplementary Fig.1d,e). Together, these findings demonstrate that Angptl4 expression in macrophages is regulated by PPARs and hypoxia.

Global ANGPTL4 deficiency attenuates atherosclerosis progression. Previous studies have reported conflicting results concerning the role of ANGPTL4 in atherogenesis ${ }^{31,40}$. To dissect the importance of ANGPTL4 during the progression of atherosclerosis, we bred Angptl4 ${ }^{-/-}$mice with the athero-prone $\mathrm{Ldlr}^{-/-}$mice. Then, Ldlr ${ }^{-/}$and Angptl4 ${ }^{-/-}$Ldlr ${ }^{-/-}$mice were fed a WD for 12 weeks. Interestingly, we observed a marked reduction in atherosclerosis in Angptl4 ${ }^{-/-} \mathrm{Ldlr}^{-/-}$mice compared with $L d l r^{-/}$mice (Fig. 2a). Moreover, macrophage accumulation was also significantly reduced in ANGPTL4deficient atherosclerotic lesions (Fig. 2a).

In contrast to the apparently beneficial effects seen in atherosclerotic plaques, Angptl4 ${ }^{-}-L_{d l r^{-I}}$ mice exhibited a severe phenotype with peritonitis, depleted fat deposition in the gut, decreased body weight and severe gut inflammation (Fig. 2b-d), consistent with previous reports of Angptl4 ${ }^{-/-}$mice fed a high-fat $\operatorname{diet}^{27,41}$. Additionally, we observed decreased food intake and survival in these mice, with almost a third mice (4 out of 13 mice) dying before 12 weeks on a WD diet. Gut cross-section analysis revealed a massive infiltration of CD68positive macrophages in the intestinal wall of Angptl4 ${ }^{-/-} \mathrm{Ldlr^{-/- }}$ mice (Fig. 2d). Not surprisingly, given the role of ANGPTL4 in inhibiting LPL, plasma TG levels in Angptl4 ${ }^{-/-} L d l r^{-/-}$were drastically reduced compared with $L d l r^{-/}$mice (Fig. 2e). Moreover, total plasma cholesterol levels and VLDL particles were also markedly reduced (Fig. 2f-h). Overall, our results show that ANGPTL4 deficiency reduces atherosclerosis; however, the extreme phenotype observed in Angptl4 ${ }^{-/}$L $d l r^{-/}$mice suggests that the reduced atherosclerosis may be a secondary effect of the detrimental changes caused by whole body depletion of ANGPTL4. These confounding observations indicate that the metabolic abnormalities observed with whole body modulation of ANGPTL4 make assessment of the role of ANGPTL4 in atherosclerosis development complicated and thus, tissue-specific ANGPTL4 modulation might mitigate these systemic effects in multiple organs and provide meaningful data.

Haematopoietic ANGPTL4 deficiency promotes atherogenesis. Given that ANGPTL4 is highly expressed in macrophage-derived foam cells, we sought to determine the role of macrophagespecific ANGPTL4 during atherogenesis. To this end, $\mathrm{Ldlr}^{-9-}$ mice were lethally irradiated and transplanted with the BM from Angptl4 ${ }^{-/-}$and WT donor mice. Reconstitution of the donor BM was confirmed by genotyping the blood (Supplementary Fig. 2). Then the mice were fed a WD for 12 weeks and atherogenesis was assessed. In contrast to the reduced atherosclerosis observed in germline ANGPTL4-deficient mice, $L d l r^{-/-}$mice transplanted with ANGPTL4-deficient BM had significantly larger atherosclerotic lesions compared with the ones with WT BM (Fig. 3a-c). Similar findings were observed in female $\mathrm{Ldlr}^{-/-}$mice transplanted with Angptl4 ${ }^{-/-}$and WT BM (Supplementary Fig. 3a). Interestingly, the accelerated atherosclerosis observed in $L d l r^{-/}$mice reconstituted with ANGPTL4-deficient BM was not associated with difference in circulating plasma lipid levels (Fig. 3d-g and Supplementary Fig. 3c,d). Moreover, we did not find any differences in gut inflammation as assessed by CD68 staining and body weight, indicating that atherogenic effect observed in mice transplanted with Angptl4 $4^{-/-} \mathrm{BM}$ is independent of systemic inflammation (Supplementary Figs $3 \mathrm{~b}$ and $4 \mathrm{a}, \mathrm{b}$ ).

In human atherosclerotic vascular disease, plaque morphology is a more important predictor of plaque disruption and acute clinical events than plaque size $e^{42}$. In particular, the size of the necrotic core of advanced plaques is a key determinant of plaque vulnerability ${ }^{43}$. Analysis of lesions for acellular non-fibrotic areas revealed an increase in plaque necrosis in mice transplanted with Angptl4 ${ }^{-/-}$BM compared with the ones with WT BM (Fig. 4a). This increase could reflect a primary increase in macrophage apoptosis. Indeed, atherosclerotic plaques isolated from $\mathrm{Ldlr}^{-/}-$ mice transplanted with Angptl4 ${ }^{-/-} \mathrm{BM}$ showed a significant increase in TUNEL-positive cells (Fig. 4b). Moreover, cellular immunostaining showed that many of the TUNEL-positive nuclei were in macrophage-rich regions (Fig. 4b). Macrophage content in the atherosclerotic plaques was similar between groups despite larger lesions observed in $L d l r^{-/-}$mice with ANGPTL4deficient BM (Fig. 4c). Recent studies have shown that local proliferation dominates lesional accumulation of macrophages in advanced atherosclerosis ${ }^{44}$. Thus, we assessed if the absence of ANGPTL4 in macrophages influences macrophage proliferation in vivo. As shown in Fig. 4d, the number of Ki-67-positive cells was similar in atherosclerotic lesions in both groups of mice. Finally, we assessed the collagen content in the lesions, which is associated with plaque stability, using picrosirius staining. Despite a difference in the necrotic core area, collagen content was similar between groups of $L d l r^{-/}$mice transplanted with WT and Angptl4 ${ }^{-\%}-$ BM (Fig. 4e). This finding might be explained by the similar levels of macrophage accumulation in the plaques of both groups of mice, as macrophages are responsible for the modulation of collagen content by releasing metalloproteinases ${ }^{45}$. Taken together, these results indicate that ANGPTL4 deficiency in macrophages enhances the progression of atherosclerosis and increases macrophage apoptosis resulting in larger necrotic core in atherosclerotic lesions.

Haematopoietic ANGPTL4 deficiency promotes leukocytosis. To appreciate the multiple mechanisms of atherogenesis in intact vessels, we analysed the quantitative expression patterns of inflammation in the vessel wall of $L d l r^{-\digamma}$ mice transplanted with WT or Angptl4 ${ }^{-/-}$BM after 12 weeks on WD by qRT-PCR. As expected by the increased atherogenesis observed in $\mathrm{Ldlr}^{-/-}$mice reconstituted with Angptl4 ${ }^{-/-}$BM, the expression levels of several pro-inflammatory molecules including, Tnf $\alpha, C c l 3$ and Il-6 and cell adhesion molecules Icam-1 and Sele were significantly upregulated compared with $L d l r^{-/-}$mice transplanted with WT BM (Fig. 5a). To determine if the difference in pro-inflammatory gene expression was due to differences in aortic cellular composition, we analysed macrophage infiltration and activation in the artery wall of $\mathrm{Ldlr}^{-/-}$ mice transplanted with WT or Angptl4 ${ }^{-/-}$BM after 12 weeks on a WD. As shown in Fig. 5b, ANGPTL4 deficiency in haematopoietic cells markedly increased the accumulation of macrophages, which were identified as Lin-CD $11 b+F 4 / 80^{\text {high }}$ cells, and inflammatory ${ }^{8}$ Ly-6C positive $\left(\mathrm{Ly}-6 \mathrm{C}^{\mathrm{hi}}\right)$ monocytes in the artery wall (Fig. 5b). These data demonstrate that ANGPTL4 deficiency in BM-derived cells results in increased vascular inflammation and elevated infiltration of monocyte/macrophages in the arterial wall of $\mathrm{Ldlr}^{-/-}$mice.

Leukocytosis, in particular monocytosis and neutrophilia, is associated with increased cardiovascular risk in humans, and develops in hypercholesterolemic atherosclerotic animal models ${ }^{46,47}$. To investigate whether there was a difference in circulating leukocytes between $L d l r^{-/-}$mice transplanted with 
a

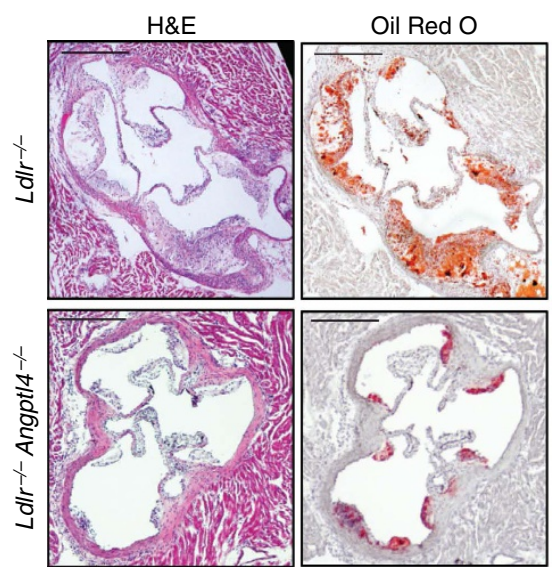

CD68 a-SMC DAPI
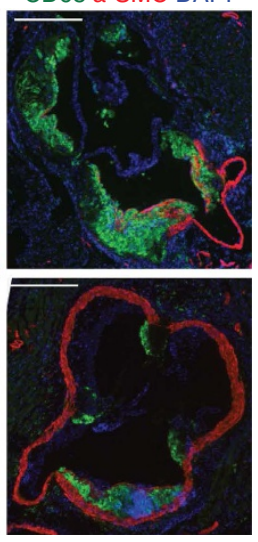
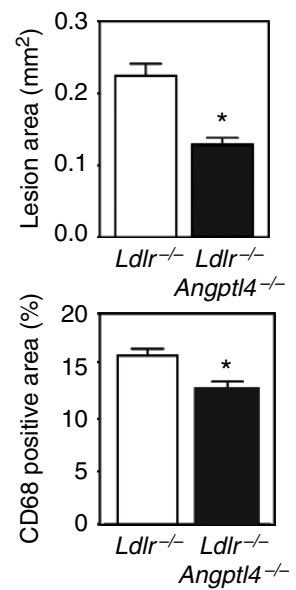

b

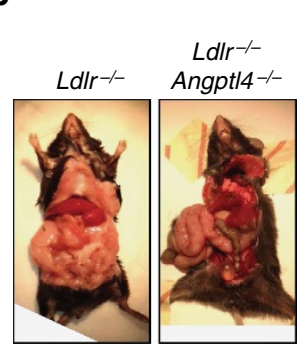

c

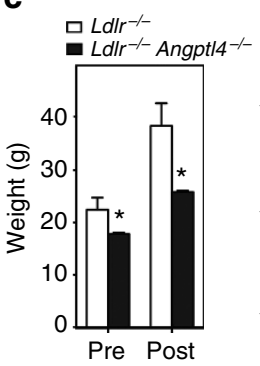

d

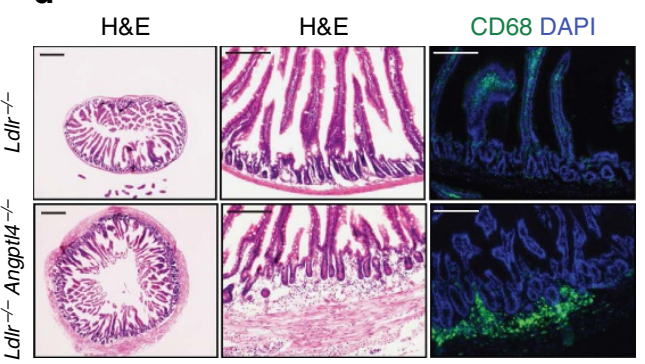

e

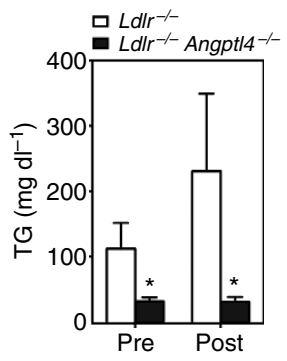

f

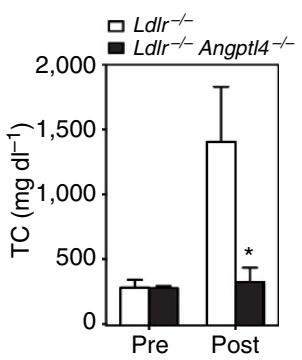

g

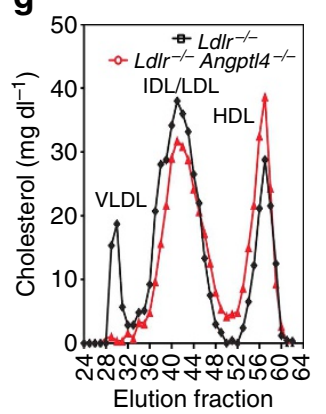

h

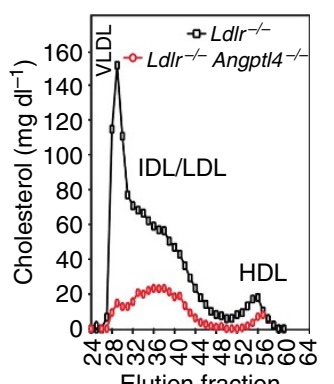

Elution fraction

Figure 2 | Absence of ANGPTL4 attenuates the progression of atherosclerosis. (a) Representative histological analysis of cross-sections of the aortic sinus from $\mathrm{Ldll}^{-/}$- and Angpt/4 ${ }^{-/} \mathrm{Ldll}^{-} /{ }^{-}$mice stained with H\&E, Oil Red O and CD68/SMC-actin/DAPI. Quantification of the lesion area and macrophage content is shown in the right panels ( $n=9$ per group). Scale bars, $400 \mu \mathrm{m}$. (b) Representative pictures of whole body $L d l r^{-/-}$and Angpt/4 ${ }^{-/}-L d l r^{-/}$mice on WD diet 12 weeks. (c) Body weight analysis of $L d l r^{-/}-$and Angpt/ $4^{-/}-L d l r^{-/}-$mice before and after WD diet $(n=9$ per

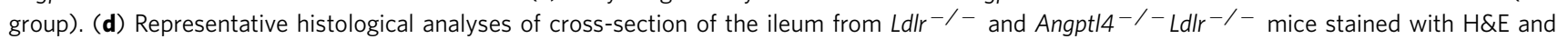
macrophage marker CD68. Scale bars, $400 \mu \mathrm{m}$. (e-h) Measurement of plasma TG (e), cholesterol (f) and lipoprotein profile from pooled plasma (g-h) of $\mathrm{Ldll}^{-{ }^{-}-}$and Angpt/4-/- $\mathrm{Ldll}^{-/-}$mice before and after 12 weeks on WD diet ( $n=9$ per group). All data are the mean \pm s.e.m.; ${ }^{\star} P<0.05$ by comparison with data from $L d l r^{-/-}$mice by unpaired $t$-test.

BM from WT and Angptl4 ${ }^{-/-}$mice, we profiled the myeloid population in the peripheral blood before and after feeding a WD. Interestingly, we found a marked increase in the proportion of inflammatory Ly-6C hi monocytes and neutrophils in the Angptl4 ${ }^{-/-}$BM transplanted group (Fig. 5c).

To determine whether the increased circulating monocytes and neutrophils reflected a difference in the HSPC proliferation, we analysed BM populations in the $L d l r^{-/-}$mice transplanted with BM from WT and Angptl4 $4^{-/-}$mice following 12 weeks of WD feeding. While there was no difference in the frequency of primitive Lin-Sca + c-Kit + (LSK) cells, there was a markedly different distribution of the immediate precursors of leukocytes, myeloid progenitors (MP), between the groups (Fig. 6a). Within the MPs, Angptl4 ${ }^{-/-}$BM transplanted mice had a significantly higher frequency of CMPs and a lower frequency of granulocyte/monocyte progenitors (GMPs) and megakaryocyte/ erythroid progenitors (MEPs) compared with WT BM transplanted mice (Fig. 6a). CMPs are the immediate precursors to GMPS, and sources of monocytes and neutrophils ${ }^{48}$. CMPs of Angptl4 ${ }^{-/}-\mathrm{BM}$ transplanted mice were highly positive for CD34, a cell surface marker for primitive haematopoietic stem cells (Fig. 6a). Taken together, these results demonstrate that ANGPTL4 deficiency in haematopoietic cells results in abnormal hematopoiesis and causes increase in CMP and leukocyte counts in $L d l r^{-/}$mice when fed a WD and contributes to the progression of atherosclerosis.

ANGPTL4 deficiency promotes CMP expansion and survival. To gain insight into the mechanism of increased progenitor populations in Angptl4 ${ }^{-/-} \mathrm{BM}$ transplanted mice, first we 
a

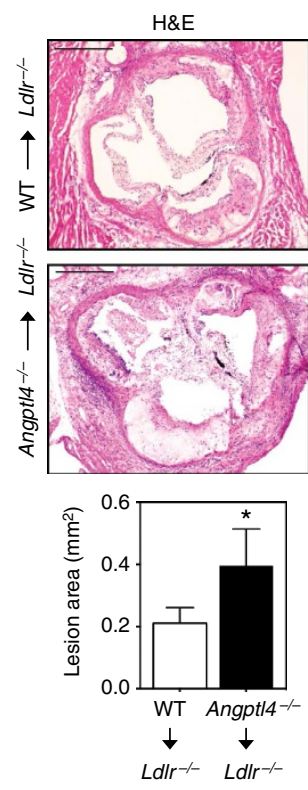

d

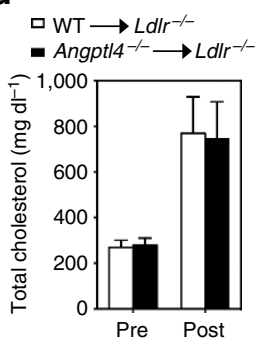

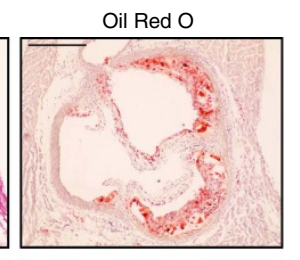
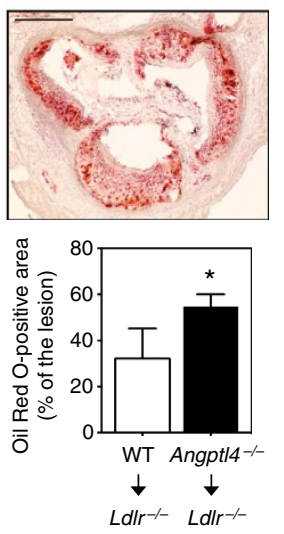

e

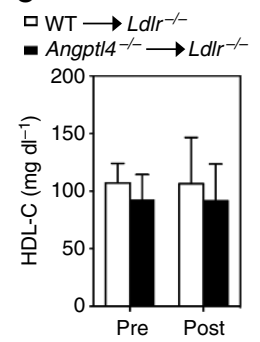

b
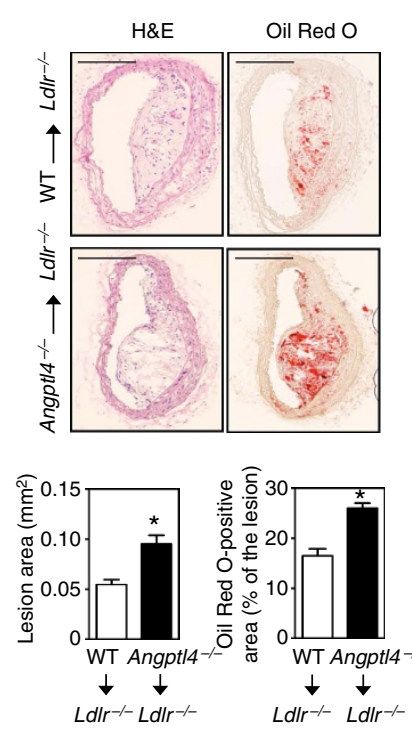

C
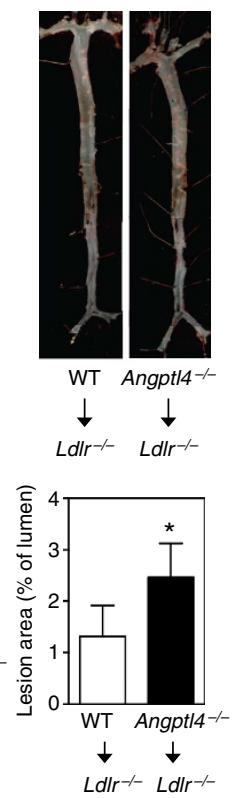

f

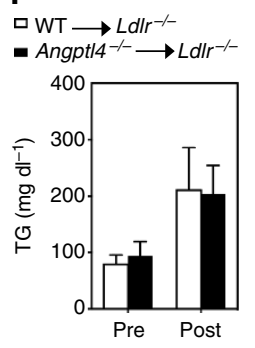

g

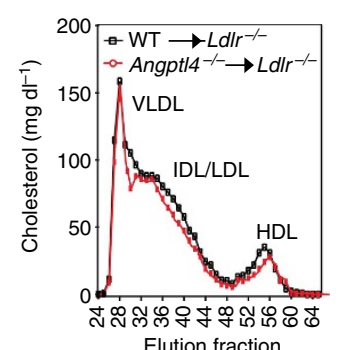

Figure 3 | Haematopoietic ANGPTL4 deficiency enhances atherogenesis. (a,b) Representative histological analysis of cross-sections of the aortic sinus (a) and brachiocephalic arteries (b) isolated from male Ldlr-/- chimeras with WT or Angpt/4-/- BM after 12 weeks of WD stained with H\&E and Oil Red O. Quantification of lesion area and Oil Red O-positive lesion area is shown in the panel below respective figure ( $n=10$ per group for aortic sinus and $n=6$ per group for brachiocephalic arteries). Scale bars, $400 \mu \mathrm{m}$. (c) Representative pictures from en face analysis of aortas from Ldlr ${ }^{-/-}$ chimeras with WT or Angpt/4-/- BM after 12 weeks on WD diet. Proportion of Oil Red O-positive area in the en face preparation is quantified in the lower panel ( $n=8$ per group). Total cholesterol level (d), HDL cholesterol level (e) and TG level (f) in the blood plasma of $L d l r^{-/}-$chimeras with WT or Angpt $/ 4^{-/-}$BM before and after 12 weeks on WD ( $n=10$ per group). (g) Lipoprotein profile from pooled plasma $\left(n=5\right.$ each group) of $L d l r^{-/-}$chimeras with WT or Angpt/4 ${ }^{-/-}$BM after 12 weeks on WD diet. All data are the mean \pm s.e.m.; ${ }^{\star} P<0.05$ by comparison with data from $L d l r^{-/}-$chimeras with WT BM by unpaired $t$-test.

checked the HSPC distribution in the BM of WT and Angptl4 -/mice. Angptl4 ${ }^{-/-}$BM had higher frequency of CMPs and lower frequency of GMPs and MEPs without any significant difference in the frequency of LSK populations, suggesting that Angptl4 ${ }^{-/-}$ animals have inherently aberrant progenitor population distribution. This was paralleled by an increase in the number of granulocyte monocyte colony-forming units (CFU-GM) and multipotent granulocyte, erythrocyte, monocyte, megakaryocyte CFUs (CFU-GEMM) that are capable of generating myeloid cells as assessed by colony-forming assays (Fig. 6b,c). Furthermore, in order to compare the proliferation potential of individual precursor cell types, CMPs and LSK cells from the WT and Angptl4 ${ }^{-/-}$BM were sorted, and single cells were used for colony-forming assays. Notably, higher numbers (62 out of 90) of Angptl4 ${ }^{-/-}$LSKs gave rise to colonies compared with WT LSKs (49 out of 90). The increase was more evident in CFU-GM and CFU-GEMM colonies (Fig. 6d). More importantly, almost twice as many Angptl4 ${ }^{-/}$CMPs (49 out of 150) were capable of giving rise to colonies compared with WT CMPs (26 out of 150) with the difference more pronounced in CFU-GM, CFU-GEMM and BFU-E (erythrocyte, burst forming unit) (Fig. 6d). These results show the ability of Angptl4 $^{-}{ }^{-}$progenitors to grow into more primitive colonies, and suggest that they are more 'stem-like' compared with WT progenitors. In contrast to the BM, there was no difference in the mobilization of the progenitor cells, as there was no difference in the number of colonies from the peripheral blood of WT and Angptl4 ${ }^{-1-}$ mice (Fig. 6f).

To determine if the increased number of colonies in Angptl4 ${ }^{-/}$was a result of increased proliferation, we assessed proliferation using $\mathrm{Ki}-67$ staining. Although the number of actively proliferating Ki-67 + CMPs tended to be higher, it failed to reach significance (Fig. $6 \mathrm{~g}$ ). To further evaluate the proliferating cells, we performed cell cycle analysis of the progenitor cells. Interestingly, significantly more CMPs but not the GMPs within Angptl4 ${ }^{-9}$ BM were in S/G2 phase confirming the earlier observations that a higher number of Angptl4 ${ }^{-/-}$CMPs were undergoing proliferation (Fig. 6h). Since almost twice the number of Angptl4 ${ }^{-}$- CMPs gave rise to colonies despite having only around $15 \%$ more cells in proliferative cell cycle, we hypothesized that Angptl4-/- CMPs have some additional features that 
a

H\&E
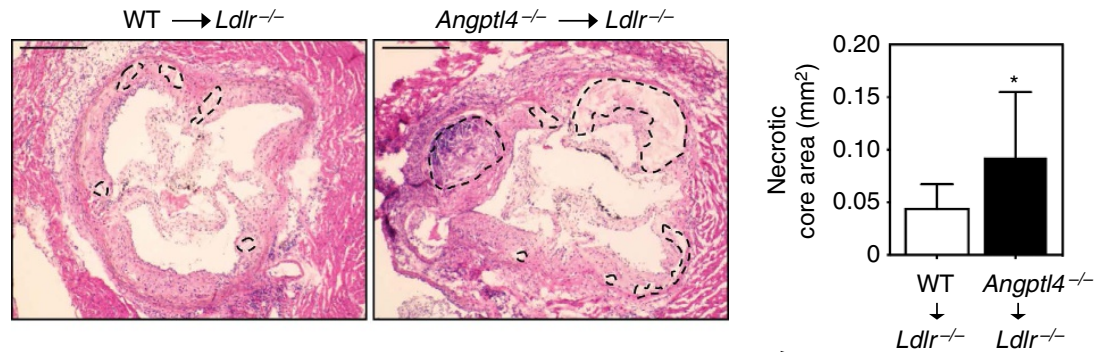

b
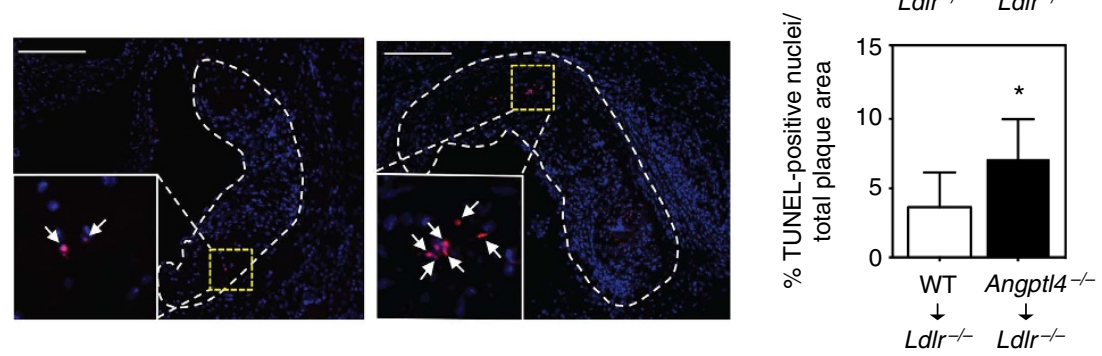

C
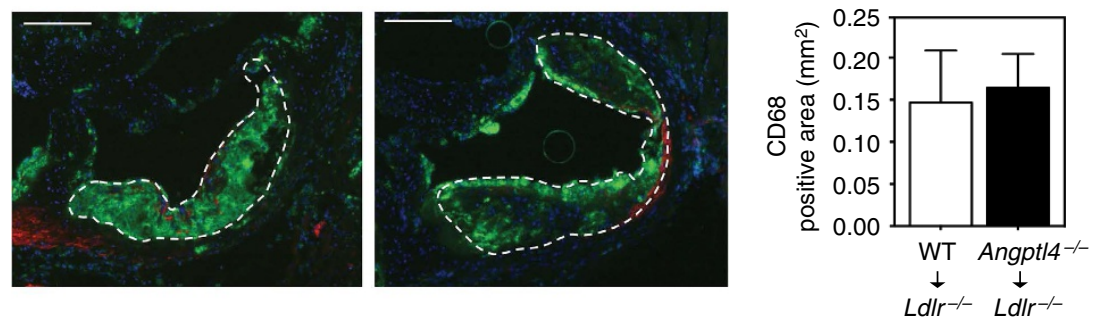

d
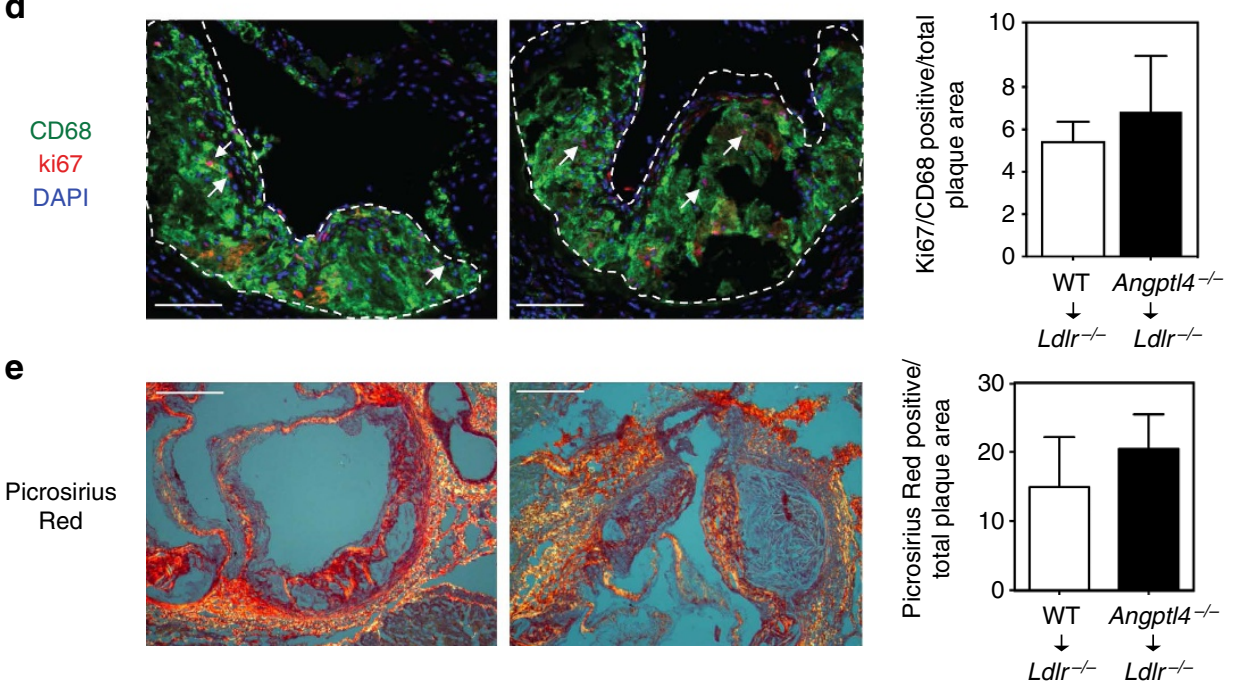

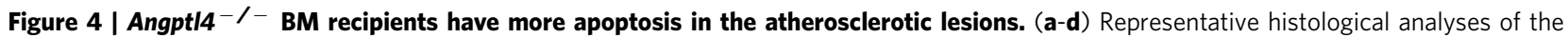
cross-sections of the aortic sinus from $\mathrm{Ldll}^{-/-}$chimeras with WT or Angpt/4-/- BM after 12 weeks on WD stained with (a) H\&E showing necrotic areas within dotted boundary (scale bars, $400 \mu \mathrm{m}$ ), (b) TUNEL; inset shows magnified TUNEL-positive area (scale bars, 200 $\mu \mathrm{m}$ ), (c) CD68/ $\alpha$-SMA/DAPI (scale bars, $200 \mu \mathrm{m}$ ), (d) CD68/Ki67/DAPI (scale bars, $200 \mu \mathrm{m}$ ) and (e) Picrosirius Red staining (scale bars, $200 \mu \mathrm{m}$ ). Total necrotic area, TUNEL-positive nuclei, macrophage content, Ki67-positive macrophages and collagen content are quantified in the right panels. $N=10$ per group for a-d and $n=6$ per group for e. All data are the mean \pm s.e.m.; ${ }^{\star} P<0.05$ by comparison with data from $L d l r^{-/}$- chimeras with WT BM by unpaired $t$-test.

supported their survival. To test this hypothesis, we assessed apoptosis among progenitor cells using Annexin V staining. Interestingly, Angptl4 ${ }^{-/-}$CMPs were significantly less apoptotic than WT CMPs (Fig. 6i). These observations suggest that the leukocytosis observed in the Angptl4 ${ }^{-/}-\mathrm{BM}$ transplanted mice is possibly an exaggeration of existing differences in the HSPC population in Angptl4 ${ }^{-1-}$ mice, whereby these mice have increased CMPs with higher proliferation potential and better survival and thus have the potential to give rise to more progenies including neutrophils and monocytes.
One of the possible mechanisms that can regulate HSPC proliferation is through signalling mediated from cell surface lipid rafts $^{49}$. For example, excessive cholesterol accumulation in ABC transporter-deficient stem cells increases lipid rafts in stem cell membranes, resulting in increased surface localization of the IL-3/GM-CSF receptor and subsequent downstream signalling and cell proliferation in response to IL-3/GM-CSF ${ }^{6}$. We assessed whether there was a difference in lipid raft content in the stem cell progenitors of WT and Angptl4 ${ }^{-/-}$mice. Remarkably, there were more lipid rafts in the Angptl4 ${ }^{-/-}$CMPs as assessed by 
a

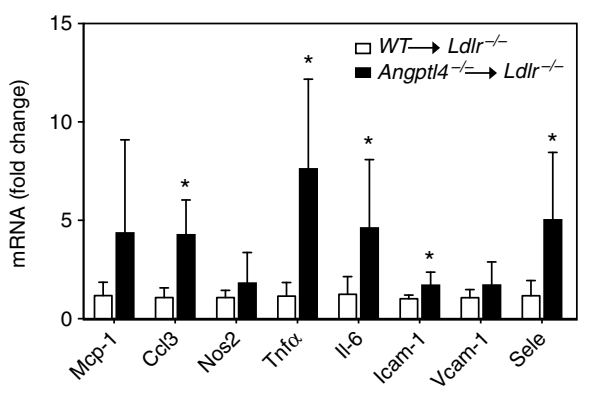

b

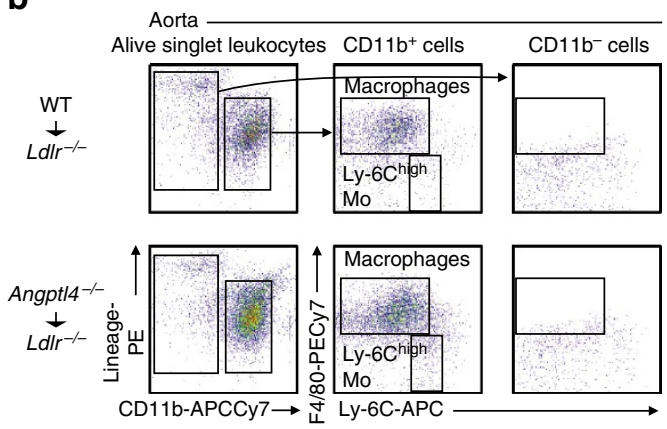

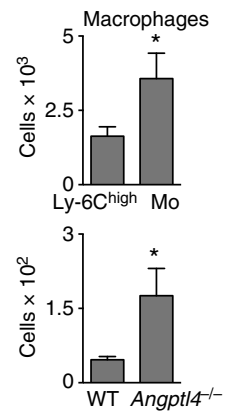

C
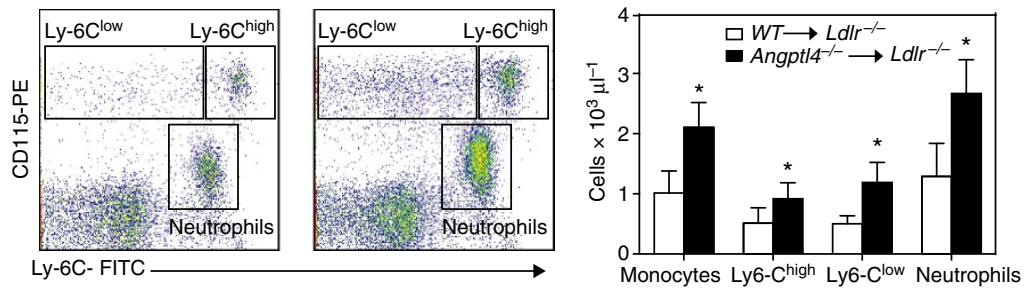

Figure 5 | Haematopoietic ANGPTL4 deficiency causes vascular inflammation and leukocytosis. (a) mRNA expression of inflammatory genes in the whole aorta of $\mathrm{Ldll}^{-/-}$chimeras with WT or Angpt/4 ${ }^{-/-}$BM after 12 weeks on WD ( $n=9$ per group). (b) Dot plots showing gating schemes of macrophages and monocytes from whole aorta of $\mathrm{Ldlr}^{-/-}$chimeras with WT or Angpt/4 ${ }^{-/-}$BM after 12 weeks on WD. (right) The quantification of total number of macrophages and Ly-6Chigh monocytes ( $n=6$ per group). (c) Representative flow cytometry plots showing monocyte and neutrophil populations from $\mathrm{Ldlr}^{-/-}$chimeras with WT or Angpt/4-/- BM after 12 weeks on WD. Total monocytes and neutrophils are quantified on the right panel by extrapolating the proportion of cells from flow cytometry to the total number of leukocytes per $\mu$ measured using Hemavet haematology analyser ( $n=10$ per group). All data are the mean \pm s.e.m.; ${ }^{\star} P<0.05$ by comparison with data from $L d l r^{-/}-$chimeras with WT BM by unpaired $t$-test.

cholera toxin $\mathrm{B}(\mathrm{CTxB})$ staining, suggesting that the difference in proliferation and survival of CMPs is associated with increased lipid raft content (Fig. 6j).

Macrophage ANGPTL4 deficiency increases foam cell formation. In order to explore additional mechanisms that could contribute to the accelerated atherosclerosis observed in Angptl4 $4^{-1-}$ BM transplanted mice, we investigated the role of ANGPTL4 in the regulation of macrophage functions. Since ANGPTL4 expression is increased in macrophage-derived foam cells, we sought to determine whether ANGPTL4 influences lipid accumulation in macrophages. Interestingly, Angptl4 ${ }^{-1-}$ macrophages accumulated significantly more neutral lipids and cholesterol compared with WT macrophages as assessed by bodipy staining and cholesterol oxidase assay respectively (Fig. 7a,b). We next investigated the mechanisms that could contribute to increased lipid accumulation in ANGPTL4-deficient macrophages. To this end, we evaluated DiI-Ox-LDL binding and uptake by flow cytometry. Absence of ANGPTL4 enhanced Ox-LDL binding and uptake (Fig. 7c,d). Moreover, there was decreased cholesterol efflux to ApoA1 in ANGPTL4-deficient macrophages stimulated with the LXR agonist T0901317 (Fig. 7e). These results suggest that both increased Ox-LDL uptake and decreased cholesterol efflux contributed to the enhanced lipid loading observed in Angptl4 ${ }^{-/-}$macrophages. Mechanistically, we found that CD36 but not SR-A1 expression levels were higher in Angptl4 ${ }^{-/-}$ macrophages compared with WT macrophages (Fig. 7f). Surprisingly, despite the reduced cholesterol efflux in Angptl4 ${ }^{-1-}$ macrophages, there was no difference in the total protein expression of ATP-binding cassette transporters ABCA1 and ABCG1, the main transporters responsible for cholesterol efflux in macrophages (Fig. 7f). This led us to investigate whether there was a difference in the distribution of ABCAl between WT and Angptl4 ${ }^{-1-}$ macrophages as the localization of ABCA1 in the cell surface is important in order to lipidate ApoA1 (ref. 50). Therefore, we checked the surface expression of ABCA1 using a biotinylation assay and immunofluorescence studies and found that there is less surface ABCA1 in Angptl4 ${ }^{-/-}$macrophages compared with WT macrophages (Fig. 7g,h). Taken together, these results demonstrate that loss of ANGPTL4 in macrophages enhances foam cell formation by regulating influx and efflux of lipoproteins and cholesterol in a reciprocal manner.

Foam cell death gives rise to large regions within plaques containing lipids and necrotic debris ${ }^{51,52}$. It is thought that free cholesterol accumulation in the endoplasmic reticulum (ER) results in ER stress-induce apoptosis ${ }^{52}$. Given our observation that ANGPTL4 deficiency promotes lipid accumulation in macrophages, we hypothesized that this could enhance macrophage apoptosis. To this end, we incubated Angptl4 $4^{-/-}$ and WT macrophages with Ac-LDL and an acetyltransferase (ACAT) inhibitor (58035), to promote free cholesterol accumulation. Consistent with our hypothesis, cholesterol loading increases apoptosis in Angptl4 ${ }^{-1-}$ macrophages assessed by Annexin V staining (Fig. 7i).

A continuum of pro- and anti-inflammatory macrophages, with extreme polarization phenotypes, M1 and M2, can be found in atherosclerotic lesions ${ }^{53}$. While M1-skewed macrophages promote inflammation and have been shown to be more abundant in the rupture-prone sections of atherosclerotic plaques, M2 macrophages are located in more stable, cell-rich areas of plaques and are associated with plaque resolution ${ }^{54,55}$. To investigate whether ANGPTL4 influences macrophage polarization, we incubated mouse BM-derived macrophages (BMDMs) with M1 or M2 polarizing cytokines, LPS/IFN $\gamma$ or IL-4, respectively. The results showed that M1 polarized Angptl4 ${ }^{-1-}$ BMDMs expressed higher mRNA levels of M1 markers including, Nos2, Cox2 and Tnfa and protein levels of COX2 compared with WT BMDMs (Supplementary Fig. 5a,b). In contrast, the expression of Ym1, a well-established M2 marker, 
a

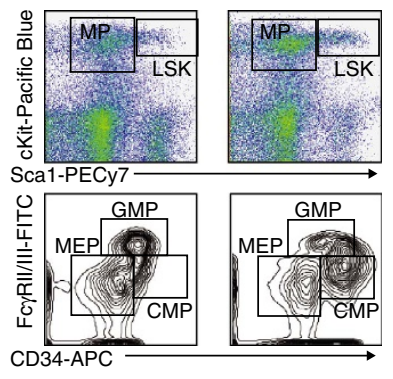

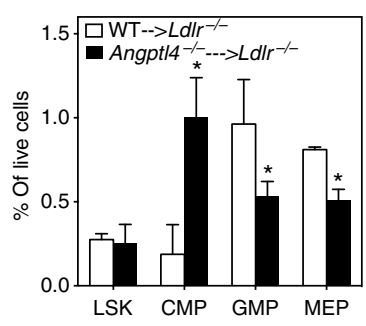

b

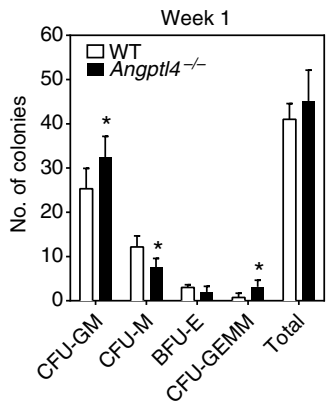

C

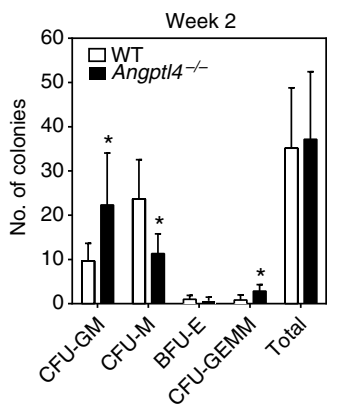

d

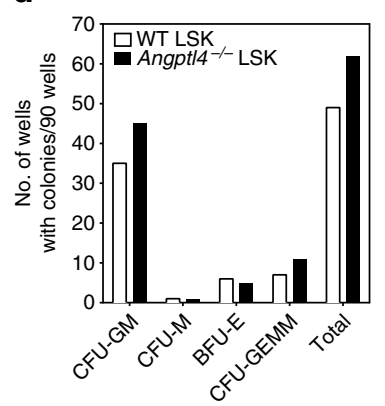

h

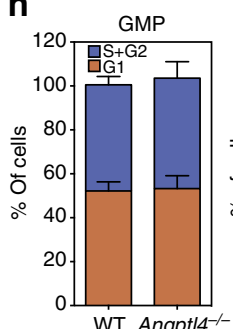

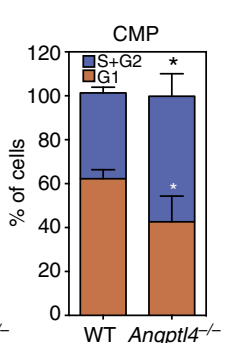

e

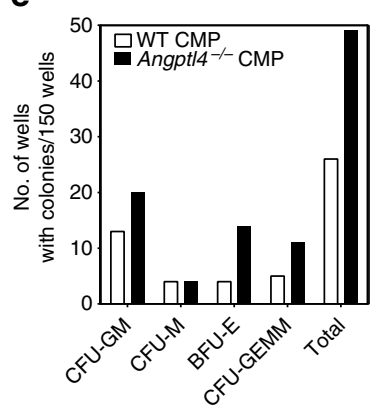

i

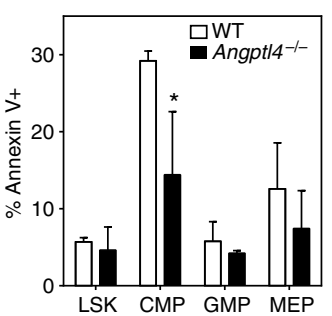

f

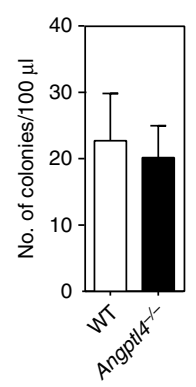

g

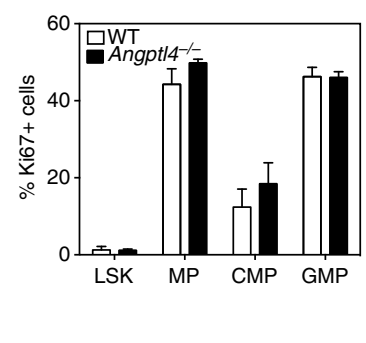

j
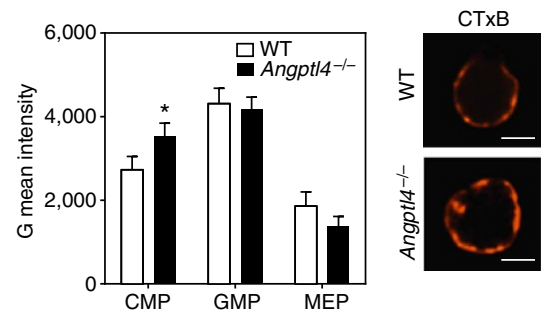

Figure 6 | Haematopoietic ANGPTL4 deficiency promotes CMP expansion. (a) Dot plots showing the gating scheme of MP and LSK cells (upper panel), contour plots showing the gating schemes of GMP, CMP and MEP cells (lower panel) in the BM of $L d l{ }^{-/-}$chimeras with WT or Angpt/4-/ - BM on WD for 12 weeks. Right panel shows the quantification these progenitor populations ( $n=5$ per group). Data are mean \pm s.e.m. ${ }^{\star} P<0.05$ by comparison with data from $L d l r^{-} /-$chimeras with WT BM by unpaired $t$-test. (b) Number of colonies from the BM isolated from WT and Angpt/4- ${ }^{-}$mice using CFU assay. Cells were plated in methylcellulose media for 1 week; colonies were counted (b), resuspended and plated for 1 more week (c) ( $n=6$ per group). Number of colony positive wells from LSKs (d) and CMPs (e) sorted from the BM and plated for 10 days individually in 96 well plates cells in methylcellulose media ( $n=4$ per group; a pool of BM was used for sorting from each group). (f) Number of colonies from $100 \mu$ l blood from WT and Angpt/4 $4^{-/}$mice plated in methylcellulose media for 10 days ( $n=3$ per group). (g) Proportion of Ki67-positive proliferating cells from indicated cell type from WT and Angpt/4-/ - BM. (h) Cell cycle analysis of GMPs and CMPs from WT and Angpt/4-/ - BM showing fraction of cells in G1 and S/G2 phase as determined by DAPI staining ( $n=5$ per group). Cell cycle phase gates were drawn as approximations of the Watson (pragmatic) cell cycle modelling algorithm. (i) Proportion of annexin V positive apoptotic cells from indicated cell types from WT and Angpt/4 ${ }^{-/-}$BM ( $n=5$ per group).

(j) Representative fluorescent images and flow cytometry quantification of lipid raft content in CMPs of WT and Angpt/4 ${ }^{-1-}$ BM assessed by CTxB staining ( $n=3$ per group). Scale bars, $5 \mu \mathrm{m}$. All data are the mean \pm s.e.m. ${ }^{\star} P<0.05$ by comparison with data from WT BM (b,c,h,i,j) by unpaired $t$-test.

was less in Angptl4 -/ BMDMs after M2 polarization, while the expression of other M2 markers was not changed (Supplementary Fig. 5a).

Monocyte/macrophage migration is an important step in the early atherogenic process. Monocytes infiltrate atherosclerotic plaques in response to cytokines, such as macrophage chemoattractant protein-1 (MCP-1, also known as CCL2 $)^{56}$. In order to investigate the role of ANGPTL4 in this process, we assessed macrophage migration in vitro. As seen in Supplementary Fig. 6a,b, MCP-1-induced migration was unaltered in macrophages lacking ANGPTL4. In agreement with this, CCR2 expression was identical in blood monocytes from $\mathrm{Ldlr}^{-/-}$mice transplanted with Angptl4 ${ }^{-/-}$and WT BM (Supplementary Fig. 6c). Collectively, these results demonstrate that in addition to increases in leukocyte count through CMP proliferation, ANGPTL4 deficiency also enhances macrophage foam cell formation, polarizes macrophages towards a M1 inflammatory phenotype, increases apoptosis susceptibility in response to cholesterol loading and promotes atherosclerosis.

\section{Discussion}

In this study, we have identified an important protective role for macrophage-derived ANGPTL4 in the pathogenesis of atherosclerosis. While global depletion of ANGPTL4 results in several confounding metabolic abnormalities and inflammation, along with attenuated atherosclerosis, haematopoietic cell-specific ablation of ANGPTL4 using BM transplantation from Angptl4 ${ }^{-/}$mice to $L d l r^{-/}$recipients results in a dramatic increase in atherosclerosis. Importantly, Angptl4 ${ }^{-/-} \mathrm{BM}$ 
a

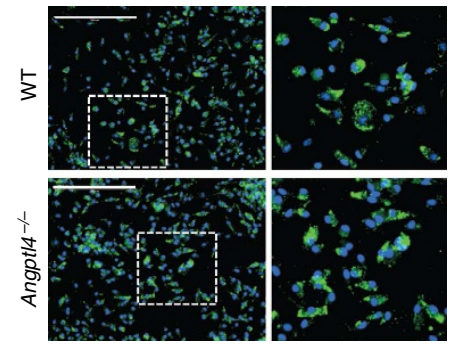

C

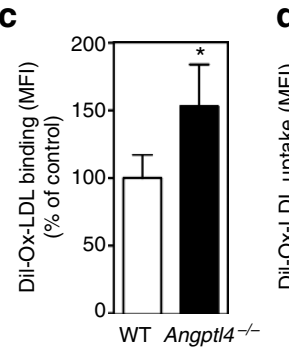

d

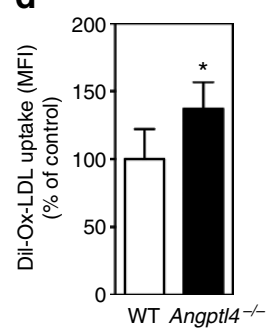

g

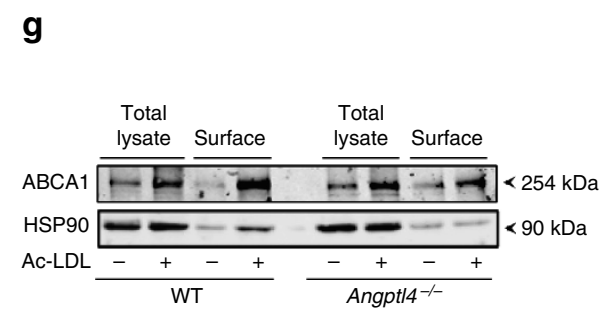

h
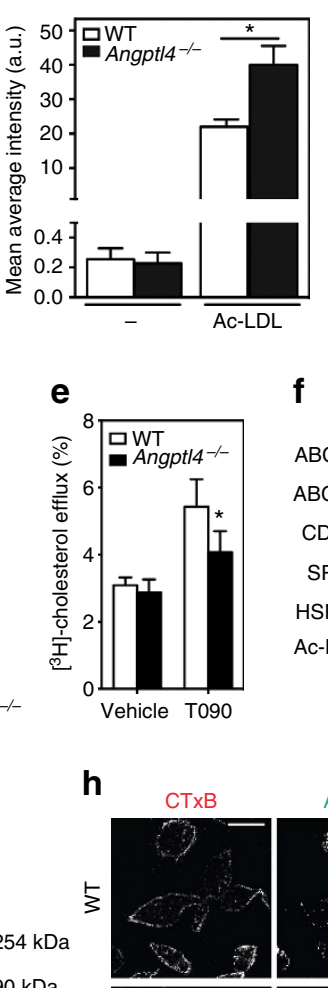

b

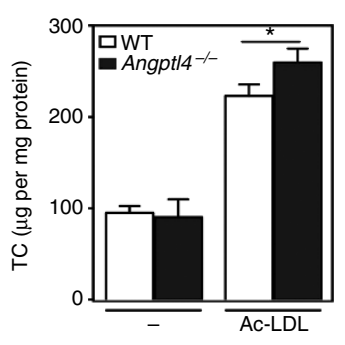

f

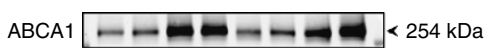

ABCG1 $-\ldots-\infty-\infty \mathrm{kDa}$

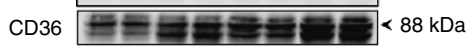

SRA $-\ldots<-\ldots 50 \mathrm{kDa}$

HSP90 $<90 \mathrm{kDa}$

Ac-LDL

$\frac{-++}{\text { WT }} \frac{-++}{\text { Angpt/4 }}$
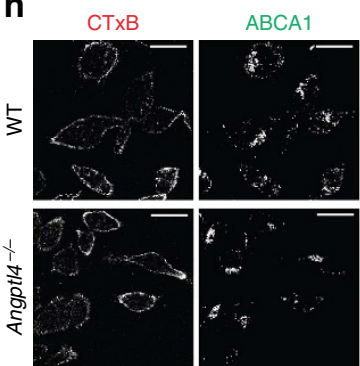

Merge/nuclei
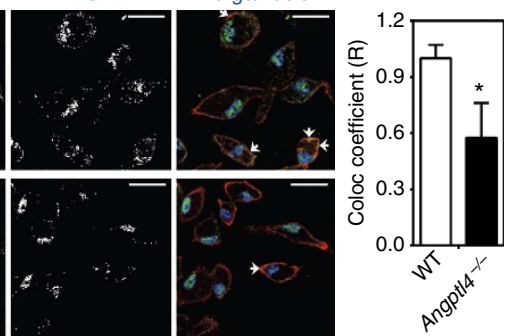

i
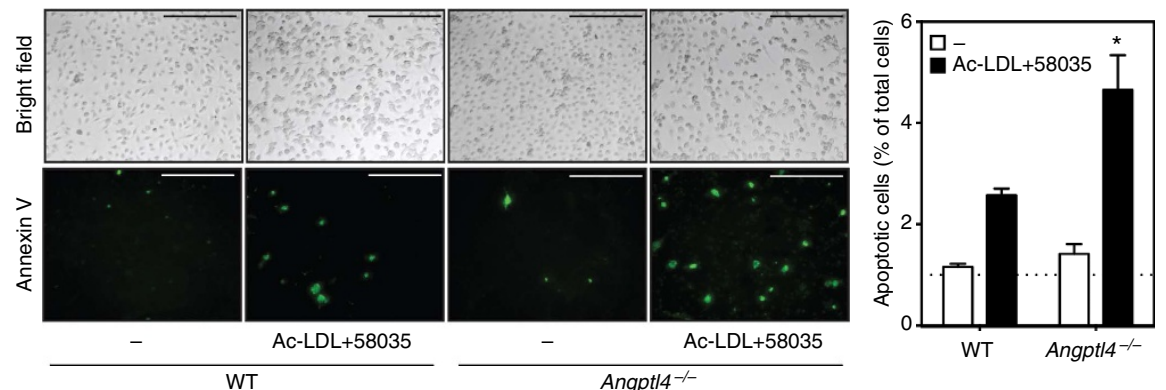

Figure 7 | ANGPTL4 deficiency promotes macrophage foam cell formation and apoptosis. (a) Representative pictures from WT and Angpt/4 ${ }^{-/}-$mouse peritoneal macrophages incubated with or without Ac- LDL $\left(120 \mu \mathrm{g} \mathrm{ml}^{-1}\right)$ for $24 \mathrm{~h}$ and stained with BODIPY $493 / 503\left(1 \mu \mathrm{g} \mathrm{ml}{ }^{-1}\right)$ and DAPI (Green and blue, respectively). Scale bar, $5 \mu \mathrm{m}$. Quantification of the mean average intensity is in the right panel. (b) Total cholesterol content in peritoneal macrophages isolated from WT and Angpt/4 ${ }^{-/}$mice incubated with or without Ac-LDL $\left(120 \mu \mathrm{g} \mathrm{ml}^{-1}\right)$ for $24 \mathrm{~h}$. (c) Flow cytometry analysis of Dil-Ox-LDL binding in peritoneal macrophages incubated with Dil-Ox-LDL ( $30 \mu \mathrm{g}$ cholesterol per ml) for 30 min at $4{ }^{\circ} \mathrm{C}$. At the end of the incubation period, cells were washed and incubated in RPMI $10 \% \mathrm{FBS}$ media for $15 \mathrm{~min}$ at $37^{\circ} \mathrm{C}$ to allow the internalization. (d) Flow cytometry analysis of Dil-Ox-LDL uptake in peritoneal macrophages incubated with Dil-Ox-LDL ( $30 \mu \mathrm{g}$ cholesterol per $\mathrm{ml}$ ) for $2 \mathrm{~h}$ at $37^{\circ} \mathrm{C}$. The results are expressed in terms of specific MFI after subtracting auto-fluorescence of cells incubated in the absence of Dil-Ox-LDL. (e) Cholesterol efflux to apolipoprotein A1 (ApoA1) in peritoneal macrophages isolated from WT and Angpt/4 ${ }^{-/-}$mice stimulated with or without T0901317 (T090). (f) Western blot analysis of indicated proteins in peritoneal macrophages from WT and Angpt/4 ${ }^{-/-}$mice incubated with or without Ac-LDL $\left(120 \mu \mathrm{g} \mathrm{ml}^{-1}\right)$ for $24 \mathrm{~h}$. (g) Western blot analysis

(representative of three blots) of $A B C A 1$ expression in $\mathrm{WT}$ and Angpt/4 ${ }^{-/-}$peritoneal macrophages incubated with Ac-LDL for $24 \mathrm{~h}$. Surface ABCA1 was isolated using biotinylation followed by incubation with neutravidin. HSP9O is used as loading control ( $\mathrm{f}$ and $\mathrm{g}$ ). Full scans of westerns blots are provided in Supplementary Fig. 8. (h) Representative confocal images of mouse peritoneal macrophages from WT and Angpt/4 $/{ }^{-}$mice incubated with Ac-LDL for $24 \mathrm{~h}$ and stained with cholera toxin B (CTxB), ABCA1 and DAPI. Quantification of co-localization of CTxB and ABCA1 is on the right panel. Scale bar, $10 \mu \mathrm{m}$. (i) Representative images of WT and Angpt/4 ${ }^{-/-}$macrophages cultured on coverslips and treated with or without $A c-L D L\left(120 \mu g ~ \mathrm{ml}^{-1}\right)$ in combination with ACAT inhibitor (58035) for $24 \mathrm{~h}$ to induce lipid-loading-induced apoptosis (scale bars, $200 \mu \mathrm{m}$ ). Apoptosis was detected using Annexin-V staining. Right panel shows the quantification of percentage of apoptotic cells from four random fields from each cover slip. All data represent the mean \pm s.e.m. from at least three experiments in duplicate; ${ }^{\star} P<0.05$ compared with WT macrophages by unpaired $t$-test. MFI, median intensity of fluorescence. 


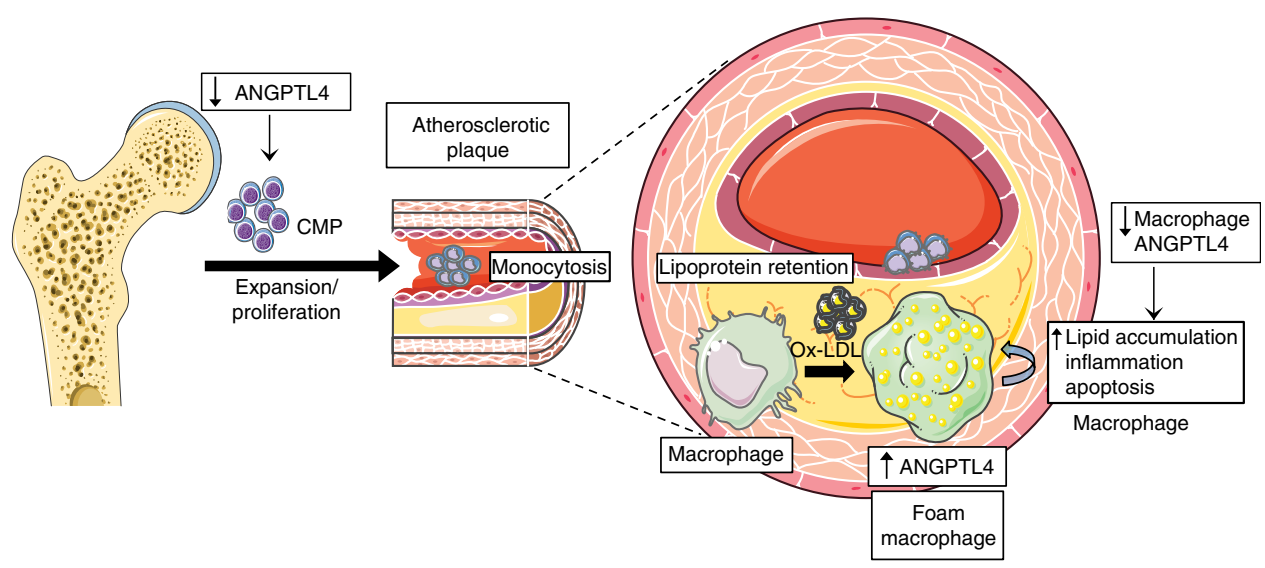

Figure 8 | ANGPTL4 deficiency results in monocytosis and massive atherosclerosis. Schematic diagram showing haematopoietic ANGPTL4 function in atherosclerosis. ANGPTL4 is induced in macrophages in response to lipoprotein loading. ANGPTL4 deficiency in macrophages results in increase in foam cell formation, inflammation and apoptosis of macrophages within atherosclerotic plaques. Concomitantly, ANGPTL4 deficiency in haematopoietic cells results in an increase in the frequency and survival of CMPs, and upon WD feeding results in an elevated level of circulating monocytes. Overall, ANGPTL4 deficiency in haematopoietic cells promotes massive atherosclerosis.

transplanted mice have elevated blood leukocyte counts, higher frequency of Ly6-C $\mathrm{C}^{\text {hi }}$ inflammatory monocytes and macrophages in aortic lesions, and increased vascular inflammation compared with mice transplanted with WT BM. Differences in leukocyte count seem to be associated with skewed progenitor population distribution, as the frequency of CMP population is higher in

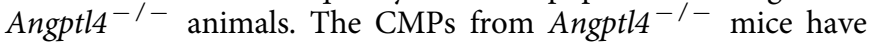
higher proliferative potential, increased lipid raft content and decreased apoptosis. This study provides a definitive role of haematopoietic ANGPTL4 during the progression of atherosclerosis by controlling leukocyte populations, and preventing lipid overloading and generation of macrophage foam cells (Fig. 8). This report demonstrates for the first time that ANGPTL4 can modulate selective HSPC proliferation, although several members of ANGPTL family with similar structures have been shown to stimulate HSPC expansion ${ }^{9,11,57,58}$.

We demonstrate that ANGPTL4 deficiency in haematopoietic cells accelerates atherosclerosis progression. Increased atherogenesis in haematopoietic ANGPTL4-deficient mice seems to have resulted from two distinct mechanisms- a higher leukocyte count and enhanced lipid loading/foam cell formation and subsequent apoptosis and inflammation. In response to hypercholesterolemia, the BM and spleen overproduce pro-inflammatory Ly- $6 \mathrm{C}^{\text {hi }}$ monocytes that can infiltrate and preferentially accumulate and differentiate to macrophages in lesions ${ }^{8,59}$. Consistent with this, we found a higher frequency of leukocytes in $\mathrm{Ldlr}^{-/-}$mice transplanted with Angptl4 ${ }^{-1}$ BM as a consequence of higher proliferation of CMPs in the BM. However, the frequency of GMPs, the immediate precursors of leukocytes, were lower in the Angptl4 ${ }^{-1-}$ BM transplanted mice. The discrepancy in the ratios of GMPs, the immediate precursors of monocytes, and CMPs, the precursors of GMPs, in ANGPTL4-deficient mice is intriguing and needs further investigation. First, as the most direct precursors of monocytes, the GMPs might be differentiating to their progeny more quickly than usual. In other words, it is possible that the low GMP number reflects a faster transition from GMP to monocytes in the animals deficient in ANGPTL4. It is similarly possible that CMPs, whose production is heightened, do not replenish GMPs quickly enough. Thus, a combination of these processes would lead to the observed phenotype. Another possibility might be that CMPs do not go through the GMP intermediate at all. The hierarchical haematopoietic tree, where one intermediate must give rise to another before finally becoming a terminally differentiated leukocyte has been challenged by several recent studies showing that upstream progenitors can bypass downstream progenitors as they differentiate to leukocytes ${ }^{60}$. Although we have no evidence that this is the case, it is possible that CMPs differentiate to monocytes directly. Further studies are warranted to elucidate the mechanisms for the selective increase in CMP population observed in ANGPTL4-deficient BM.

Although previous studies have reported that ANGPTL4 prevents lipid overload in macrophages ${ }^{27}$, a comprehensive understanding of the mechanisms behind this process and subsequent impact on atherosclerosis has been lacking. We provide experimental evidence showing that ANGPTL4 promotes foam cell formation by concomitantly increasing modified LDL uptake and decreasing cholesterol efflux. The increase in uptake seems to be mediated through at least two mechanisms- increased CD36 expression and inhibition of LPL activity. We found that CD36 expression is higher in Angptl4 ${ }^{-/-}$macrophages and is further enhanced with Ac-LDL. CD36 expression is regulated by modified LDL particles and free fatty acids ${ }^{61}$. As such, enhanced lipid loading in Angptl4 $4^{-/-}$macrophages seems to follow a positive feedback response, whereby increased CD36 on the cell surface leads to more lipoprotein loading and fatty acid release and this in turn leads to increased CD36 expression. LPL is also known to facilitate retention and uptake of lipoproteins by cell surface receptors through its bridging activity ${ }^{62}$. In fact, macrophage-specific overexpression of LPL results in the accumulation of macrophage foam cells and atherosclerotic lesion formation, whereas LPL deletion results in the opposite $^{28,63}$. Since ANGPTL4 is a strong inhibitor of LPL, enhanced modified LDL uptake in ANGPTL4-deficient macrophages might also be mediated through increased activity of LPL. However, the fact that transgenic mice expressing catalytically active or inactive LPL in macrophages show similar atherogenic effects suggests that the bridging function of LPL is sufficient to drive its effect in macrophages ${ }^{64}$. In a recent study, ANGPTL4 treatment resulted in more pronounced reduction in Ox-LDL uptake by macrophages compared with Orlistat, an inhibitor of LPL activity, indicating that ANGPTL4 possibly also regulates non-catalytic bridging activity of $\mathrm{LPL}^{31}$. Further investigation using LPL and ANGPTL4-deficient macrophages is required to confirm this possibility.

In addition to a significant reduction in Ox-LDL uptake, we also demonstrate a marked decrease in cholesterol efflux in Angptl $4^{-1-}$ macrophages. Importantly, we found that absence of 
ANGPTL4 influences ABCA1 expression in the cell surface. Although it is not clear how the absence of ANGPTL4 influences the cellular localization of ABCA1, a previous report has shown that elevated unsaturated fatty acids such as palmitoleate and oleate, which are enriched in macrophage foam cells ${ }^{65}$, decrease ABCA1 in plasma membrane ${ }^{66}$. Thus, it is possible that absence of ANGPTL4 could increase intracellular unsaturated fatty acid content leading to a reduction in ABCA1 cell surface expression.

In summary, this study demonstrates that ANGPTL4 can have profound effects on quantitative and qualitative properties of macrophages. On the one hand, ANGPTL4 appears to regulate the net blood monocyte content in lipid-rich conditions by restricting the expansion of precursors of monocytes, CMPs. On the other hand, ANGPTL4 suppresses lipid overloading in macrophages and prevents generation of foam cells and inflammation. Altogether, by controlling these critical myeloid features, haematopoietic ANGPTL4 reduces the progression of atherosclerosis. The findings from this study suggest that interventions capable of enhancing ANGPTL4 expression, specifically in macrophages, may lessen plaque progression.

\section{Methods}

Animals. Male C57BL/6 (WT) and $\mathrm{Ldlr}^{-/-}$mice were purchased from Jackson Laboratories (Bar Harbor, ME) and kept under constant temperature and humidity in a $12 \mathrm{~h}$ controlled dark/light cycle. Angptl ${ }^{-/-}$mice were provided by the laboratory of Andras Nagy (Samuel Lunenfeld Research Institute, Mount Sinai Hospital). Angptl4 ${ }^{-/-}$mice that have been backcrossed eight generations onto a C57BL/6 background were crossed with $\mathrm{Ldlr}^{-1-}$ mice, also on the C57BL/6 background, so that mice heterozygous at both loci could be generated. These Angptl4 $4^{+/}-\mathrm{Ldlr}^{+/-}$mice were crossed a second time with $\mathrm{Ldlr^{-/- }}$ mice. The Angptl4 $4^{+/} \mathrm{Ldlr}^{-/-}$progeny from this round of breeding were then intercrossed, producing Angptl4 ${ }^{-/} \mathrm{Ldlr}^{-/-}$and Angptl4 ${ }^{+/+} \mathrm{Ldlr}^{-{ }^{-}}$littermates that were used as controls for all studies. Male mice were used for experiments after they were $8-10$ weeks old. Accelerated atherosclerosis was induced by feeding the mice for 12 weeks with a WD containing $1.25 \%$ cholesterol (ResearchDiets, D12108). All the experiments were approved by the Institutional Animal Care Use Committee of New York University and Yale University School of Medicine.

mRNA microarray analysis. Mouse thioglycollate-elicited peritoneal macrophages we incubated in presence of Ac-LDL $\left(120 \mu \mathrm{g} \mathrm{ml}^{-1}\right)$ for $24 \mathrm{~h}$. Total RNA was extracted using TRIzol (Invitrogen), and mRNA was purified using and mRNA isolation Kit (Qiagen). The purity and integrity of total RNA sample was verified using the Agilent Bioanalyzer (Agilent Technologies, Santa Clara, CA). mRNA was hybridized to Illumina expression profiling microarray (Mouse WG-6 v2.0 Expression beads Chip) according to the manufacturer's directions. The data discussed in this publication have been deposited in NCBÍs Gene Expression Omnibus and are accessible through GEO Series accession number GSE83090 (https://www.ncbi.nlm.nih.gov/geo/query/acc.cgi?acc=GSE3090). Raw data were normalized and analysed by GeneSpring GX software version 11.5 (Agilent Technologies)

Lipids and lipoprotein profile measurements. Mice were fasted for $12-14 \mathrm{~h}$ before blood samples were collected by retro-orbital venous plexus puncture. Then, plasma was separated by centrifugation and stored at $-80^{\circ} \mathrm{C}$. Total plasma cholesterol and TGs were enzymatically measured (Wako Chemicals, USA) according to the manufacture's instructions. The lipid distributions in plasma lipoprotein fractions were assessed by fast-performed liquid chromatography gel filtration with 2 superose 6 HR 10/30 columns (Pharmacia).

Histology and morphometric analyses. Mouse hearts were perfused with $10 \mathrm{ml}$ of PBS and were put in $10 \mathrm{ml}$ of $4 \%$ paraformaldehyde overnight. After incubation in PFA, hearts were washed with PBS and left with PBS for $1 \mathrm{~h}$. Next, hearts were put in $30 \%$ sucrose until the next day. Finally, hearts were embedded in OCT and frozen. Serial sections were cut at $8 \mu \mathrm{m}$ thickness using a cryostat. Every third slide from the serial sections was stained with haematoxylin and eosin (H\&E) and each consecutive slide was stained with oil red $\mathrm{O}(\mathrm{ORO})$ for quantification of lesion area. Aortic lesion size of each animal was obtained by averaging the lesion areas in four sections from the same mouse. The necrotic core area was measured as a percentage of the total plaque area from the three sections from the same mouse. Collagen content was assessed by Picrosirius red-staining of consecutive slides from serial sections. CD68 staining (1:200; Serotec; \#MCA1957) was used as a macrophage marker using consecutive slides from serial sections. Apoptotic cells in lesions were detected by TUNEL after proteinase K treatment, using in situ Cell Death Detection kit, TMR red (Roche) according to the manufacturer's instructions. Nuclei were counterstained with DAPI for $10 \mathrm{~min}$. The data are expressed as the number of TUNEL-positive cells per $\mathrm{mm} 2$ cellular lesion area. Proliferating cells in each lesion were detected by Ki-67 staining (1:100; Abcam; \#66155). Percentage of proliferating cells was calculated as the number positive Ki-67-labelled nuclei divided by the number of DAPI-stained nuclei. In separate sections from $\mathrm{Ldlr}^{-1-}$ mice on high-cholesterol diet, ANGPTL4 (1:100; Invitrogen; \#40-9800) and CD68 expression were detected using immunofluoresecence.

Human atherosclerotic plaques. Ten atherosclerotic plaques (stages V-VI) from patients undergoing carotid endarterectomy in our institution were fixed with paraformaldehyde and embedded in paraffin for histological analysis. In addition, 10 carotid endarterectomy samples were dissected separating the stenosis CP from the non-complicated (NCP) fibrous adjacent area as previously described ${ }^{62}$. The $\mathrm{CP}$ was defined as the lesion, usually localized at the origin of the internal carotid artery responsible for the surgery. The $\mathrm{CP}$ contained an important proportion of inflammatory cells (Stary stages V-VI), whereas the NCP adjacent areas were mainly composed of VSMCs (Stary stage III). These tissues were snap frozen in liquid nitrogen and homogenates were divided and resuspended for mRNA and protein analysis. The study was approved by the hospital's ethics committee (IIS-Fundación Jiménez Díaz) according to the institutional and the Good Clinical Practice guidelines and was performed in accordance with the Declaration of Helsinki. Written informed consent was obtained from patients undergoing carotid endarterectomy and samples were kept under the IIS-FJD biobank supervision [Ministerio de Sanidad y Consumo, Instituto de Salud Carlos III, biobancos (RD09/0076/00101)].

Immunohistochemistry of carotid endarterectomy plaques. The carotid atherosclerotic plaques were fixed with PFA and embedded in paraffin for histological analysis and were then cross-sectioned into $4 \mu \mathrm{m}$ thick pieces, dewaxed, and rehydrated. Immunohistochemical staining was assessed using anti-CD68 (1:250; Dako; clone kp1, M0814), or anti- ANGPTL4 (1:100; Thermo Scientific; \#PA5-26216) antibodies, followed by mouse secondary antibody. ABComplex/HRP (Vector Laboratories) was added, and sections were stained with 3,3'-diaminobenzidine (Dako). Corresponding hematoxylin staining was used for nucleus identification. Negative controls using the corresponding IgG were included for checking nonspecific staining.

Bone marrow transplantation. Eight-week-old male $\mathrm{Ldlr}^{-/-}$mice were lethally irradiated with double dose of 550 rads $(5.5 \mathrm{~Gy})$ from a caesium source $4 \mathrm{~h}$ apart before transplantation. BM was collected from femurs of donor Angptl4 ${ }^{-1-}$ or WT mice by flushing with sterile medium (RPMI 1640, $2 \%$ fetal bovine serum (FBS), $5 \mathrm{U} \mathrm{ml}^{-1}$ heparin, $50 \mathrm{U} \mathrm{ml}^{-1}$ penicillin and $50 \mu \mathrm{g} \mathrm{ml}^{-1}$ streptomycin). Each recipient mouse was injected with $2 \times 10^{6} \mathrm{BM}$ cells through retro-orbital injection. Four weeks after BM transplantation, peripheral blood was collected by retro-orbital venous plexus puncture for PCR analysis of BM reconstitution. For atherosclerosis study, mice were fed with WD for 12 weeks beginning 4 weeks after BM transplantation. At the end of 12 weeks, mice were killed and lipid analysis and atherosclerotic lesion analysis were performed.

Cell culture and treatments. Mouse macrophages (RAW 264.7) cells were obtained from American Type Tissue Collection (ATTC TIB-71). RAW 264.7 were grown in DMEM supplemented with $10 \%$ FBS and $1 \%$ penicillin-streptomycin, and L-glutamine. Peritoneal macrophages from adult male C57BL/6J mice were harvested by peritoneal lavage four days after intraperitoneal injection of thioglycollate $(3 \% \mathrm{w} / \mathrm{v})$. Cells were plated in RPMI 1640 supplemented with

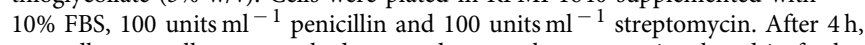
non-adherent cells were washed out, and macrophages were incubated in fresh medium containing DMEM, 10\% FBS, and 20\% L-929 cells conditioned medium for $24 \mathrm{~h}$ and cells were maintained in culture as an adherent monolayer. Peritoneal macrophages were used for different experiments.

For BMDMs isolation, cells were flushed out from femur and tibia of WT and Angptl4 ${ }^{-1-}$ mice, filtered with $40 \mu \mathrm{m}$ cell strainer (BD Falcon) and resuspended at 6-8 $\times 10^{6} \mathrm{cells} \mathrm{ml}^{-1}$ in IMDM media (Invitrogen) supplemented with $10 \%$ FBS at room temperature (RT). The cell suspension was then layered over lympholyte $\mathrm{M}$ solution (Cedarlane) at the proportion of $1 \mathrm{ml}$ per $10^{6}$ cells. After centrifugation at $1300 \mathrm{~g}$ for $20 \mathrm{~min}$ at RT, mononuclear cells were collected from the interface. Cells were washed in $\mathrm{Ca}^{2+}$ - and $\mathrm{Mg}^{2+}$-free PBS and resuspended in medium containing Iscove's DMEM, 20\% FBS, 20\% L-929 cells conditioned medium and $0.25 \mu \mathrm{g} \mathrm{ml}^{-1}$ fungizone and plated at a density of $1.7 \times 10^{5}$ cells per $\mathrm{ml}$ on non-tissue coated plates. After 6 days in culture, non-adherent cells were eliminated and adherent cells were harvested for experiments.

Cell migration assay. Cell migration assays were performed with a modified Boyden chamber with Costar Trans-well inserts (Corning). The inserts were coated with a solution of $0.1 \%$ gelatin. Sub-confluent BMDMs from WT and Angptl4 $4^{-1}$ mice were serum starved for $6 \mathrm{~h}$. Solutions of MCP-1 $\left(100 \mathrm{ng} \mathrm{ml}^{-1}\right)$, M-CSF (100 ng ml ${ }^{-1}$ ), and $0.1 \%$ BSA were prepared in Dulbecco's modified Eagle's medium (DMEM) and added to the bottom chambers. Macrophages 
$\left(7.5 \times 10^{4}\right.$ cells) were added to the upper chambers. After $16 \mathrm{~h}$ incubation at $37^{\circ} \mathrm{C}$, cells on both sides of the membrane were fixed and stained with the Diff-Quik staining kit (Baxter Health). Cells on the upper side of the membrane were removed with a cotton swab. The average number of cells per field on the lower side of the membrane from four high-power $(\times 400)$ fields was counted. Macrophage chemotaxis was also quantified using a Real-time Cell Invasion and Migration (RT-CIM) $\times$ CELLigence assay system with monitoring every $5 \mathrm{~min}$ (Roche Applied Science). Cell migration in response to MCP-1 (100 $\mathrm{ng} \mathrm{ml}^{-1}$ ) was measured till $20 \mathrm{~h}$

Cellular cholesterol measurement and foam cell formation assays.

Thioglycollate-elicited peritoneal macrophages from WT and Angptl4 ${ }^{-/-}$ mice were plated on 12-well plates and incubated with or without Ac-LDL $\left(120 \mu \mathrm{g} \mathrm{ml}^{-1}\right)$. After $24 \mathrm{~h}$, intracellular cholesterol content was measured using the Amplex Red Cholesterol Assay Kit (Molecular Probes, Life Technologies), according to the manufacturer's instructions. Cholesterol ester was estimated by subtracting free cholesterol from total cholesterol. For the foam cell formation assay, peritoneal macrophages were grown on glass coverslips and treated with or without Ac-LDL $\left(120 \mu \mathrm{g} \mathrm{ml}^{-1}\right)$ for $24 \mathrm{~h}$. Cells were then fixed with $4 \%$ PFA for $20 \mathrm{~min}$. After washing three times with $1 \times \mathrm{PBS}$, cells were blocked with $1 \%$ BSA in PBS for $30 \mathrm{~min}$ and stained with BODIPY 493/503 (Life Technologies) for $30 \mathrm{~min}$ at RT. Next, cells were washed twice with 1x PBS and stained the nuclei with DAPI for $10 \mathrm{~min}$ to visualize the nuclei. Finally, cells were mounted on glass slides with Prolong-Gold (Life Technologies). From each cover slip, four random fields were selected and images were acquired using Zeiss Axiovert $2000 \mathrm{M}$ fluorescence microscope (Carl Zeiss). All gains for the acquisition of comparable images were maintained constant. Analysis of different images was performed using ImageJ (NIH) and Adobe Photoshop CS5.

Cholesterol efflux assays. Peritoneal macrophages from WT and Angptl4 ${ }^{-1-}$ mice were seeded at a density of $1 \times 10^{6}$ cells per well 1 day before loading with $0.5 \mu \mathrm{Ci}$ per ml $\left[{ }^{3} \mathrm{H}\right]$-cholesterol for $24 \mathrm{~h}$ with or without T0901317 $\left(3 \mu \mathrm{moll}^{-1}\right)$ for $12 \mathrm{~h}$. Cells were then washed twice with PBS and incubated in RPMI 1640 medium supplemented with $2 \mathrm{mg} \mathrm{ml}^{-1}$ fatty acid free bovine serum albumin in the presence acetyl-coenzyme A ACAT inhibitor $\left(2 \mu \mathrm{moll}^{-1}\right.$; Novartis Corporation, New York, NY, USA) for $4 \mathrm{~h}$ before the addition of $50 \mu \mathrm{g} \mathrm{ml}^{-1}$ human ApoA1 in FAFA media. Supernatants were collected after $6 \mathrm{~h}$ and expressed as a percentage of $\left[{ }^{3} \mathrm{H}\right]$-cholesterol in the media per total cell $\left[{ }^{3} \mathrm{H}\right]$-cholesterol content (total effluxed $\left[{ }^{3} \mathrm{H}\right]$-cholesterol + cell-associated $\left[{ }^{3} \mathrm{H}\right]$-cholesterol).

Apoptosis assay. For cell death assay, mouse peritoneal macrophages were grown on coverslips and incubated with Ac- LDL $\left(120 \mu \mathrm{g} \mathrm{ml}^{-1}\right)$ and ACAT inhibitor $\left(58035,10 \mu \mathrm{g} \mathrm{ml}^{-1}\right)$. After $24 \mathrm{~h}$, cells were washed with Annexin V buffer and stained with FITC-conjugated Annexin V (1:20, Biolegend) and DAPI for $15 \mathrm{~min}$ in dark. From each cover slip, four random fields were selected and images were captured using Zeiss Axiovert $2000 \mathrm{M}$ fluorescence microscope (Carl Zeiss). Quantification of the images was performed using Image J.

Luciferase assay. PPRE in the intron 3 of Angptl4 initially cloned into SEAP vector (gift of Sander Kersten, Wageningen University, The Netherlands) was excised out and cloned into pGL3 vector (Promega) downstream of firefly luciferase reporter gene. Raw macrophages were co- transfected with pGL3 construct (400 ng per well) and Renilla luciferase control plasmid (phRL-CMV; Promega, $1.5 \mathrm{ng}$ per well) in 24-well plates using Lipofectamine 2000 (Invitrogen) according to the manufacturer's guide. $24 \mathrm{~h}$ after the transfection, cells were treated with or without Ac-LDL or rosiglitazone (a synthetic PPAR- $\gamma$ agonist) for another $24 \mathrm{~h}$. Luciferase levels were measured using Dual Luciferase Reporter Assay System (Promega). Firefly luciferase activity was normalized to the corresponding Renilla luciferase activity and plotted as a percentage of the control. Experiments were performed in triplicate and repeated at least three times.

Blood leukocytes and aortic cells analysis. Blood was collected by retro-orbital puncture in heparinized micro-hematocrit capillary tubes. Erythrocytes were lysed with ACK lysis buffer ( $155 \mathrm{mM}$ Ammonium Chloride, $10 \mathrm{mM}$ Potassium Bicarbonate, $0.01 \mathrm{mM}$ EDTA, pH 7.4). WBC were resuspended in 3\% FBS in PBS, blocked with $2 \mu \mathrm{g} \mathrm{ml}^{-1}$ of FcgRII/III, then stained with a cocktail of antibodies. Monocytes were identified as CD115hi and subsets as Ly6-Chi and Ly6-Clo; neutrophils were identified as CD115 ${ }^{\mathrm{lo}} \mathrm{Ly} 6-\mathrm{C}^{\mathrm{hi}} \mathrm{Ly} 6-\mathrm{G}^{\mathrm{hi}}$. The following antibodies were used (all from BioLegend): FITC-Ly6-C (AL-21), PE-CD115 (AFS98), APC- Ly6-G (1A8). All antibodies were used at 1:300 dilutions.

For the analysis of leukocytes in aortic tissue, entire aorta was perfused with PBS, cut in small pieces and subjected to enzymatic digestion with $400 \mathrm{U} \mathrm{ml}^{-1}$ collagenase I, $125 \mathrm{U} \mathrm{ml}^{-1}$ collagenase XI, $60 \mathrm{U} \mathrm{ml}^{-1}$ DNase I and $60 \mathrm{U} \mathrm{ml}^{-1}$ hyaluronidase (Sigma-Aldrich) for $1 \mathrm{~h}$ at $37^{\circ} \mathrm{C}$ while shacking. Total viable cell numbers were obtained using Trypan Blue (Cellgro).
Haematopoietic stem cells analysis. BM was harvested from femurs and tibias. Cells were blocked with $2 \mu \mathrm{g} \mathrm{ml}^{-1}$ of FITC-conjugated FcgRII/III and stained for lineage markers (B220 (RA3-6B2), CD11b (M1/70), Gr-1 (RB6-8C5), TER- 119, CD8a- (536.7), CD4 (RM4-5), IL7R (A7R34), all APC-Cy7-conjugated), BV421-ckit (2B8), PE-Cy7-Scal (D7), APC-CD34 (RAM34). CMPs were identified as Lin-/ckit + Sca1-/CD34int, FcgRII/IIIint, while GMP as Lin-/ckit + Scal-/CD34int, FcgRII/IIIhi. All the antibodies were from BioLegend and antibody dilutions were 1:300-1:500. Flow cytometry was performed using a BD LSRII and flow cytometry and imaging were performed using Amnis Imagestream-X MarkII imaging flow cytometer. All flow cytometry data was analysed using FlowJo software (Tree Star) and imaging from flow cytometry was analysed using IDEAS application software. Apoptotic cells were analysed using Annexin V staining (1:20, Biolegend) and proliferative cells were identified using Ki-67 (1:300, Biolegend) and DAPI stainings.

GM-CFU assay. BM was isolated from the femur of WT and Angptl4 ${ }^{-1-}$ mice. Total of $2 \times 10^{4} \mathrm{BM}$ cells were plated in $1 \mathrm{ml}$ methylcellulose-based media that was supplemented with an assortment of recombinant cytokines (Methocult GF M3434; StemCell Technologies) in $35 \mathrm{~mm}$ culture dishes. The number of GM-CFUs per dish was counted after 1 week, colonies were resuspended and $2 \times 10^{4}$ cells from the suspension were replated in fresh methylcellulose-based media. Colonies were counted after another week in the media. In another set of experiments, CMP, GMP and LSK cells were sorted from the BM of WT and Angptl4 ${ }^{-/-}$mice using BD FACSAria cell sorter. Before sorting, HSPCs were enriched using mouse progenitor cell enrichment kit (Stem Cell Technologies). Identification scheme for different progenitor population for sorting was similar to the one used above to analyse progenitor cells. Single cells from LSK and CMPs were plated in 96 well plates in methylcellulose-based media. Total number of wells with colonies and types of colonies were evaluated after 10 days.

Dil-Ox-LDL uptake and binding assays. DiI-Ox-LDL lipoproteins were oxidized and labelled with the fluorescent probe DiI (Molecular Probes, Invitrogen). For the uptake assays, mouse peritoneal macrophages were washed once in IX PBS and incubated in fresh media containing DiI-Ox-LDL ( $30 \mu \mathrm{g}$ cholesterol per $\mathrm{ml}$ ) for $2 \mathrm{~h}$ at $37^{\circ} \mathrm{C}$. For the binding assays, cells were incubated for $15 \mathrm{~min}$ at $4{ }^{\circ} \mathrm{C}$ to stop membrane internalization. Then, cells were treated with fresh media containing Dil-Ox-LDL ( $30 \mu \mathrm{g}$ cholesterol per $\mathrm{ml})$ for $1 \mathrm{~h}$ and $30 \mathrm{~min}$ at $4{ }^{\circ} \mathrm{C}$. At the end of the incubation period, cells were washed and $1 \mathrm{ml}$ of RPMI $10 \%$ FBS added for $15 \mathrm{~min}$ at $37^{\circ} \mathrm{C}$ to allow the internalization. Finally, in both assays, cells were washed and resuspended in $1 \mathrm{ml}$ of PBS and analysed by flow cytometry (FACScalibur, Becton Dickinson). The results are expressed in terms of specific median intensity of fluorescence (M.I.F.) after subtracting auto-fluorescence of cells incubated in the absence of DiI-LDL.

M1 and M2 polarization assay. BMDMs from WT and Angptl4 ${ }^{-/-}$mice were stimulated for $8 \mathrm{~h}$ with IL-4 $\left(15 \mathrm{ng} \mathrm{ml}^{-1}\right)$ (R\&D) and LPS $\left(10 \mathrm{ng} \mathrm{ml}^{-1}\right.$ ) (Sigma) plus IFN $\gamma\left(20 \mathrm{ng} \mathrm{ml}^{-1}\right)(\mathrm{R} \& D)$ to polarize macrophages to M2 or M1, respectively. At the end of the treatment, cells were extensively washed with 1x PBS and RNA and protein were isolated.

Western blotting. Tissue or cells were lysed in ice-cold buffer containing $50 \mathrm{mM}$ Tris-HCl, pH 7.5, $125 \mathrm{mM} \mathrm{NaCl}, 1 \% \mathrm{NP}-40,5.3 \mathrm{mM} \mathrm{NaF}, 1.5 \mathrm{mM} \mathrm{NaP}, 1 \mathrm{mM}$ orthovanadate and $1 \mathrm{mg} \mathrm{ml}^{-1}$ of protease inhibitor cocktail (Roche) and $0.25 \mathrm{mg} \mathrm{ml}^{-1}$ AEBSF (Roche). Tissue or cell lysates were rotated at $4{ }^{\circ} \mathrm{C}$ for $1 \mathrm{~h}$ before the insoluble material was removed by centrifugation at $12000 \mathrm{~g}$ for $10 \mathrm{~min}$ After normalizing for equal protein concentration, cell lysates were resuspended in SDS sample buffer before separation by SDS-polyacrylamide gel electrophoresis. Following overnight transfer of the proteins onto nitrocellulose membranes, membranes were probed using the following antibodies (catalogue numbers and dilutions used are indicated within parentheses): rabbit polyclonal antibodies against ANGPTL4 (\#40-9800; 1:100), ABCG1 (\#NB400-132; 1:1000), COX-2 (\#160106; 1:1000) and iNOS (\#2982; 1:1000) were obtained from Invitrogen, Novus, Cayman and Cell Signaling Technology respectively; a mouse monoclonal antibody against ABCA1 (clone AB.H10; \# ab18180, 1:1000) and a rabbit polyclonal antibody against CD36 (\#ab124515; 1:1000) were obtained from Abcam; a goat polyclonal antibody against SR-A (\#sc-20444; 1:1000) was from Santa Cruz and mouse monoclonal antibodies against HSP-90 (clone 68/HSP90; \#610419, 1:1000) and GAPDH (clone GT239; \#GTX627408, 1:5000) were purchased from BD Biosciences and GeneTex, respectively. Blots were then washed and incubated with fluorescently labelled secondary antibodies (Invitrogen). Protein bands were visualized using the Odyssey Infrared Imaging System (LI-COR Biotechnology). Densitometry analysis of the gels was carried out using ImageJ software from the NIH (http://rsbweb.nih.gov/ij/).

Biotinylation of cell surface proteins. Biotinylation of surface protein was performed as explained before. Briefly, thioglycollate-elicited peritoneal macrophages from WT and Angptl4 ${ }^{-1-}$ mice were plated on six-well plates and incubated with or without Ac-LDL $\left(120 \mathrm{\mu g} \mathrm{ml}^{-1}\right)$ for $24 \mathrm{~h}$. Cells were then washed with PBS ++ 
$\left(1 \times\right.$ PBS supplemented with $0.02 \mathrm{mM} \mathrm{CaCl}_{2}$ and $\left.0.15 \mathrm{mM} \mathrm{MgCl}_{2}\right)$ and incubated for $30 \mathrm{~min}$ on ice with $250 \mu \mathrm{M}$ EZ-link SulfoNHS-SS Biotin diluted in PBS ++ (Life Technologies). Cells were again washed with $\mathrm{PBS}^{++}$and the reaction was quenched for 30 min on ice in quenching buffer $(\mathrm{PBS}++$ supplemented with $100 \mathrm{mM}$ glycine). Cells were scraped and $1 / 5^{\text {th }}$ of suspension were set aside as whole lysate and rest of the biotin-modified proteins were immunoprecipitated with NeutrAvidin agarose beads (Life Technologies) overnight at $4{ }^{\circ} \mathrm{C}$. Biotinmodified proteins were collected by centrifugation at $5,000 \mathrm{~g}$ for $5 \mathrm{~min}$. Intracellular, unmodified proteins were collected from the supernatant of the 5,000 g spin. The streptavidin beads were washed three times in $\mathrm{PBS}^{+}+$before proteins were removed from the beads by incubation at $42{ }^{\circ} \mathrm{C}$ for $20 \mathrm{~min}$, in $2 \times$ SDS sample loading buffer supplemented with $\beta$-mercaptoethanol. Biotinylated cell surface $A B C A 1$ was detected by immunoblotting total surface proteins against $A B C A 1$ antibody (1:1000; Abcam; clone AB.H10; \# ab18180).

Fluorescence microscopy. For ABCA1 localization experiments, thioglycollateelicited peritoneal macrophages were plated on coverslips and incubated with AcLDL $\left(120 \mu \mathrm{g} \mathrm{ml}^{-1}\right)$ for $24 \mathrm{~h}$. At the end of incubation, cells were washed with PBS and incubated with fluorescently labelled cholera toxin B (CTxB) in $0.1 \%$ BSA in PBS at $4{ }^{\circ} \mathrm{C}$ for $20 \mathrm{~min}$. Cells were then fixed with $4 \%$ PFA on ice for $10 \mathrm{~min}$, permeabilized with $100 \%$ pre-chilled methanol at $-20^{\circ} \mathrm{C}$ for $2 \mathrm{~min}$, blocked with $4 \%$ goat serum in PBS for $1 \mathrm{~h}$ and stained with monoclonal antibody against ABCA1 (1:1000; Abcam; clone AB.H10; \# ab18180) overnight. After three washes with $1 \times$ PBS, cells were incubated with Alexa Fluor 488 donkey anti-mouse secondary antibody (1:250, life technologies; \# A21202) for $1 \mathrm{~h}$ at RT and coverslips were mounted on glass slides with mounting media with DAPI (Vectashield, Vector Laboratories; \# H-1200). All images were analysed using a confocal microscope (Leica SP5 II) equipped with a 63X Plan Apo Lenses. All gains for the acquisition of comparable images were maintained at a constant level. Analysis of different images was performed using ImageJ (NIH) and Adobe Photoshop CS5.

RNA isolation and quantitative real-time PCR. Total RNA from human plaques or mouse aortas was isolated using the Bullet Blender Homogenizer (Next Advance) in TRIzol reagent (Invitrogen) according to the manufacturer's protocol. Total RNA from BMDM was isolated using TRIzol reagent. $1 \mu \mathrm{g}$ of total RNA was reverse transcribed using the iScript RT Supermix (Bio-Rad), following the manufacturer's protocol. Quantitative real-time PCR was performed in triplicate using SsoFast EvaGreen Supermix (BioRad) on a Real-Time Detection System (Eppendorf). The mRNA level was normalized to $18 \mathrm{~s}$ as a housekeeping gene (Primers, Supplementary Table 1).

Statistical analysis. Animal sample size for each study was chosen on the basis of literature documentation of similar well-characterized experiments $s^{67,68}$. The number of animals used in each study is listed in the figure legends and in the main text. No inclusion or exclusion criteria were used and studies were not blinded to investigators or formally randomized. In vitro experiments were routinely repeated at least three times unless otherwise noted. All data are expressed as mean \pm s.e.m. Statistical differences were measured using an unpaired two-sided Student's $t$-test. Normality was checked using the Kolmogorov-Smirnov test. A nonparametric test (Mann-Whitney) was used when data did not pass the normality test. A value of $P \leq 0.05$ was considered statistically significant. Data analysis was performed using GraphPad Prism Software Version 5.0a (GraphPad, San Diego, CA).

Data availability. All relevant data are available from the authors. The microarray data have been deposited in the NCBI Gene Expression Omnibus data base GSE83090.

\section{References}

1. Ross, R. Atherosclerosis-an inflammatory disease. N. Engl. J. Med. 340, 115-126 (1999).

2. Glass, C. K. \& Witztum, J. L. Atherosclerosis. The road ahead. Cell 104, 503-516 (2001).

3. Weijenberg, M. P., Feskens, E. J. \& Kromhout, D. White blood cell count and the risk of coronary heart disease and all-cause mortality in elderly men. Arterioscler. Thromb. Vasc. Biol. 16, 499-503 (1996).

4. Averill, L. E., Meagher, R. C. \& Gerrity, R. G. Enhanced monocyte progenitor cell proliferation in bone marrow of hyperlipemic swine. Am. J. Pathol. 135, 369-377 (1989).

5. Feldman, D. L., Mogelesky, T. C., Liptak, B. F. \& Gerrity, R. G. Leukocytosis in rabbits with diet-induced atherosclerosis. Arterioscler. Thromb. 11, 985-994 (1991).

6. Yvan-Charvet, L. et al. ATP-binding cassette transporters and HDL suppress hematopoietic stem cell proliferation. Science 328, 1689-1693 (2010).

7. Murphy, A. J. et al. ApoE regulates hematopoietic stem cell proliferation, monocytosis, and monocyte accumulation in atherosclerotic lesions in mice. J. Clin. Invest. 121, 4138-4149 (2011).
8. Swirski, F. K. et al. Ly-6Chi monocytes dominate hypercholesterolemiaassociated monocytosis and give rise to macrophages in atheromata. J. Clin. Invest. 117, 195-205 (2007).

9. Zhang, C. C. et al. Angiopoietin-like proteins stimulate ex vivo expansion of hematopoietic stem cells. Nat. Med. 12, 240-245 (2006).

10. Zhang, H. et al. HIF-1-dependent expression of angiopoietin-like 4 and L1CAM mediates vascular metastasis of hypoxic breast cancer cells to the lungs. Oncogene 31, 1757-1770 (2012).

11. Zheng, J. et al. Inhibitory receptors bind ANGPTLs and support blood stem cells and leukaemia development. Nature 485, 656-660 (2012).

12. Blank, U. et al. Angptl4 maintains in vivo repopulation capacity of CD34+ human cord blood cells. Eur. J. Haematol. 89, 198-205 (2012).

13. Sukonina, V., Lookene, A., Olivecrona, T. \& Olivecrona, G. Angiopoietin-like protein 4 converts lipoprotein lipase to inactive monomers and modulates lipase activity in adipose tissue. Proc. Natl Acad. Sci. USA 103, 17450-17455 (2006).

14. Xu, A. et al. Angiopoietin-like protein 4 decreases blood glucose and improves glucose tolerance but induces hyperlipidemia and hepatic steatosis in mice. Proc. Natl Acad. Sci. USA 102, 6086-6091 (2005).

15. Gealekman, O. et al. Enhanced angiogenesis in obesity and in response to PPARgamma activators through adipocyte VEGF and ANGPTL4 production. Am. J. Physiol. Endocrinol. Metab. 295, E1056-E1064 (2008).

16. Padua, D. et al. TGFbeta primes breast tumors for lung metastasis seeding through angiopoietin-like 4. Cell 133, 66-77 (2008).

17. Goh, Y. Y. et al. Angiopoietin-like 4 interacts with integrins betal and beta5 to modulate keratinocyte migration. Am. J. Pathol. 177, 2791-2803 (2010).

18. Yoshida, K., Shimizugawa, T., Ono, M. \& Furukawa, H. Angiopoietin-like protein 4 is a potent hyperlipidemia-inducing factor in mice and inhibitor of lipoprotein lipase. J. Lipid. Res. 43, 1770-1772 (2002).

19. Mandard, S. et al. The fasting-induced adipose factor/angiopoietin-like protein 4 is physically associated with lipoproteins and governs plasma lipid levels and adiposity. J. Biol. Chem. 281, 934-944 (2006).

20. Koster, A. et al. Transgenic angiopoietin-like (angptl)4 overexpression and targeted disruption of angptl4 and angptl3: regulation of triglyceride metabolism. Endocrinology 146, 4943-4950 (2005).

21. Romeo, S. et al. Population-based resequencing of ANGPTL4 uncovers variations that reduce triglycerides and increase HDL. Nat. Genet. 39, 513-516 (2007).

22. Talmud, P. J. et al. ANGPTL4 E40K and T266M: effects on plasma triglyceride and HDL levels, postprandial responses, and CHD risk. Arterioscler. Thromb. Vasc. Biol. 28, 2319-2325 (2008).

23. Yoon, J. C. et al. Peroxisome proliferator-activated receptor gamma target gene encoding a novel angiopoietin-related protein associated with adipose differentiation. Mol. Cell Biol. 20, 5343-5349 (2000).

24. Kersten, S. et al. Characterization of the fasting-induced adipose factor FIAF, a novel peroxisome proliferator-activated receptor target gene. J. Biol. Chem. 275, 28488-28493 (2000).

25. Koliwad, S. K. et al. Angiopoietin-like 4 (ANGPTL4, fasting-induced adipose factor) is a direct glucocorticoid receptor target and participates in glucocorticoid-regulated triglyceride metabolism. J. Biol. Chem. 284, 25593-25601 (2009).

26. Belanger, A. J. et al. Hypoxia up-regulates expression of peroxisome proliferator-activated receptor gamma angiopoietin-related gene (PGAR) in cardiomyocytes: role of hypoxia inducible factor 1alpha. J. Mol. Cell. Cardiol. 34, 765-774 (2002).

27. Lichtenstein, L. et al. Angptl4 protects against severe proinflammatory effects of saturated fat by inhibiting fatty acid uptake into mesenteric lymph node macrophages. Cell Metab. 12, 580-592 (2010).

28. Wilson, K., Fry, G. L., Chappell, D. A., Sigmund, C. D. \& Medh, J. D. Macrophage-specific expression of human lipoprotein lipase accelerates atherosclerosis in transgenic apolipoprotein e knockout mice but not in C57BL/6 mice. Arterioscler. Thromb. Vasc. Biol. 21, 1809-1815 (2001).

29. Babaev, V. R. et al. Macrophage lipoprotein lipase promotes foam cell formation and atherosclerosis in vivo. J. Clin. Invest. 103, 1697-1705 (1999).

30. Adachi, H. et al. Angptl4 deficiency decreases serum triglyceride levels in low-density lipoprotein receptor knockout mice and streptozotocin-induced diabetic mice. Biochem. Biophys. Res. Commun. 409, 177-180 (2011).

31. Georgiadi, A. et al. Overexpression of angiopoietin-like protein 4 protects against atherosclerosis development. Arterioscler. Thromb. Vasc. Biol. 33, 1529-1537 (2013).

32. Suzuki, H. et al. A role for macrophage scavenger receptors in atherosclerosis and susceptibility to infection. Nature 386, 292-296 (1997).

33. Kunjathoor, V. V. et al. Scavenger receptors class A-I/II and CD36 are the principal receptors responsible for the uptake of modified low density lipoprotein leading to lipid loading in macrophages. J. Biol. Chem. 277, 49982-49988 (2002).

34. Staiger, H. et al. Muscle-derived angiopoietin-like protein 4 is induced by fatty acids via peroxisome proliferator-activated receptor (PPAR)-delta and is of metabolic relevance in humans. Diabetes 58, 579-589 (2009). 
35. Desvergne, B. \& Wahli, W. Peroxisome proliferator-activated receptors: nuclear control of metabolism. Endocr. Rev. 20, 649-688 (1999).

36. Marsch, E., Sluimer, J. C. \& Daemen, M. J. Hypoxia in atherosclerosis and inflammation. Curr. Opin. Lipidol. 24, 393-400 (2013).

37. Lewis, J. S., Lee, J. A., Underwood, J. C., Harris, A. L. \& Lewis, C. E. Macrophage responses to hypoxia: relevance to disease mechanisms. J. Leukoc. Biol. 66, 889-900 (1999).

38. Murata, M. et al. Hypoxia upregulates the expression of angiopoietin-like-4 in human articular chondrocytes: role of angiopoietin-like- 4 in the expression of matrix metalloproteinases and cartilage degradation. J. Orthop. Res. 27, 50-57 (2009).

39. Li, H. et al. Hypoxia-inducible factor 1 alpha-activated angiopoietin-like protein 4 contributes to tumor metastasis via vascular cell adhesion molecule-1/integrin betal signaling in human hepatocellular carcinoma. Hepatology 54, 910-919 (2011).

40. Adachi, H. et al. Angptl 4 deficiency improves lipid metabolism, suppresses foam cell formation and protects against atherosclerosis. Biochem. Biophys. Res. Commun. 379, 806-811 (2009).

41. Desai, U. et al. Lipid-lowering effects of anti-angiopoietin-like 4 antibody recapitulate the lipid phenotype found in angiopoietin-like 4 knockout mice. Proc. Natl Acad. Sci. USA 104, 11766-11771 (2007).

42. Falk, E., Shah, P. K. \& Fuster, V. Coronary plaque disruption. Circulation 92, 657-671 (1995).

43. Kolodgie, F. D. et al. Pathologic assessment of the vulnerable human coronary plaque. Heart 90, 1385-1391 (2004).

44. Robbins, C. S. et al. Local proliferation dominates lesional macrophage accumulation in atherosclerosis. Nat. Med. 19, 1166-1172 (2013).

45. Newby, A. C. Metalloproteinase expression in monocytes and macrophages and its relationship to atherosclerotic plaque instability. Arterioscler. Thromb. Vasc. Biol. 28, 2108-2114 (2008).

46. Tall, A. R., Yvan-Charvet, L., Westerterp, M. \& Murphy, A. J. Cholesterol efflux: a novel regulator of myelopoiesis and atherogenesis. Arterioscler. Thromb. Vasc. Biol. 32, 2547-2552 (2012).

47. Soehnlein, O. \& Swirski, F. K. Hypercholesterolemia links hematopoiesis with atherosclerosis. Trends Endocrinol. Metab. 24, 129-136 (2013).

48. Iwasaki, H. \& Akashi, K. Myeloid lineage commitment from the hematopoietic stem cell. Immunity 26, 726-740 (2007)

49. Jahn, T., Leifheit, E., Gooch, S., Sindhu, S. \& Weinberg, K. Lipid rafts are required for Kit survival and proliferation signals. Blood 110, 1739-1747 (2007).

50. Neufeld, E. B. et al. Cellular localization and trafficking of the human ABCA1 transporter. J. Biol. Chem. 276, 27584-27590 (2001).

51. Tabas, I. Consequences of cellular cholesterol accumulation: basic concepts and physiological implications. J. Clin. Invest. 110, 905-911 (2002).

52. Kellner-Weibel, G. et al. Effects of intracellular free cholesterol accumulation on macrophage viability: a model for foam cell death. Arterioscler. Thromb. Vasc. Biol. 18, 423-431 (1998).

53. Leitinger, N. \& Schulman, I. G. Phenotypic polarization of macrophages in atherosclerosis. Arterioscler. Thromb. Vasc. Biol. 33, 1120-1126 (2013).

54. Bouhlel, M. A. et al. PPARgamma activation primes human monocytes into alternative M2 macrophages with anti-inflammatory properties. Cell Metab. 6, 137-143 (2007).

55. Stoger, J. L. et al. Distribution of macrophage polarization markers in human atherosclerosis. Atherosclerosis 225, 461-468 (2012).

56. Tedgui, A. \& Mallat, Z. Cytokines in atherosclerosis: pathogenic and regulatory pathways. Physiol. Rev. 86, 515-581 (2006).

57. Zhang, C. C., Kaba, M., Iizuka, S., Huynh, H. \& Lodish, H. F. Angiopoietin-like 5 and IGFBP2 stimulate ex vivo expansion of human cord blood hematopoietic stem cells as assayed by NOD/SCID transplantation. Blood 111, 3415-3423 (2008).

58. Khoury, M. et al. Mesenchymal stem cells secreting angiopoietin-like-5 support efficient expansion of human hematopoietic stem cells without compromising their repopulating potential. Stem Cells Dev. 20, 1371-1381 (2011).

59. Tacke, F. et al. Monocyte subsets differentially employ CCR2, CCR5, and CX3CR1 to accumulate within atherosclerotic plaques. J. Clin. Invest. 117, 185-194 (2007)

60. Notta, F. et al. Distinct routes of lineage development reshape the human blood hierarchy across ontogeny. Science 351, aab2116 (2016).
61. Tontonoz, P., Nagy, L., Alvarez, J. G., Thomazy, V. A. \& Evans, R. M. PPARgamma promotes monocyte/macrophage differentiation and uptake of oxidized LDL. Cell 93, 241-252 (1998).

62. Merkel, M. et al. Catalytically inactive lipoprotein lipase expression in muscle of transgenic mice increases very low density lipoprotein uptake: direct evidence that lipoprotein lipase bridging occurs in vivo. Proc. Natl Acad. Sci. USA 95, 13841-13846 (1998).

63. Babaev, V. R., Patel, M. B., Semenkovich, C. F., Fazio, S. \& Linton, M. F. Macrophage lipoprotein lipase promotes foam cell formation and atherosclerosis in low density lipoprotein receptor-deficient mice. J. Biol. Chem 275, 26293-26299 (2000).

64. Gustafsson, M. et al. Retention of low-density lipoprotein in atherosclerotic lesions of the mouse: evidence for a role of lipoprotein lipase. Circ. Res. 101, 777-783 (2007).

65. Spann, N. J. et al. Regulated accumulation of desmosterol integrates macrophage lipid metabolism and inflammatory responses. Cell 151, 138-152 (2012).

66. Wang, Y. \& Oram, J. F. Unsaturated fatty acids inhibit cholesterol efflux from macrophages by increasing degradation of ATP-binding cassette transporter A1. J. Biol. Chem. 277, 5692-5697 (2002).

67. Rotllan, N. et al. Hematopoietic Akt2 deficiency attenuates the progression of atherosclerosis. FASEB J. 29, 597-610 (2015).

68. Fazio, S. et al. Increased atherosclerosis in LDL receptor-null mice lacking ACAT1 in macrophages. J. Clin. Invest. 107, 163-171 (2001).

\section{Acknowledgements}

We thank Dr. Sander Kersten for generously providing the ANGPTL4 PPRE plasmid, Dr Kathryn Moore for providing $C d 36^{-/-}$mice and Dr Jordan Pober for providing the human peripheral blood mononuclear cells (PBMCs). We thank Leigh Goedeke and Juan Francisco Aranda for technical assistance and Leigh Goedeke and Nathan Price for editing the manuscript. Yale Keck Biotechnology Microarray Resource Laboratory. This work was supported by grants from the National Institutes of Health (R01HL107953 and R01HL106063 to CF-H; R01HL105945 to Y.S.), the American Heart Association (12POST9780016 to C.M.R.; 16GRNT26420047 to Y.S.), the Howard Hughes Medical Institute International Student Research Fellowship (to E.A.), and the Foundation Leducq Transatlantic Network of Excellence in Cardiovascular Research (to C.F.-H.).

\section{Author contributions}

B.A., C.F.-H. and Y.S. conceived and designed the study. B.A., N.R., E.A., C.M.R., A.W., S.H., B.G.C., A.M.F., J.M.-M. and NW performed experiments and analysed the data. J.L.M.-V., F.S., Y.S., and C.F.-H. assisted with experimental design and data interpretation. B.A. and C.F.-H. wrote the manuscript, which was commented on by all authors.

\section{Additional information}

Supplementary Information accompanies this paper at http://www.nature.com/ naturecommunications

Competing financial interests: The authors declare no competing financial interests.

Reprints and permission information is available online at http://npg.nature.com/ reprintsandpermissions/

How to cite this article: Aryal, B. et al. ANGPTL4 deficiency in hematopoietic cells promotes monocyte expansion and atherosclerosis progression. Nat. Commun. 7:12313 doi: $10.1038 /$ ncomms12313 (2016).

This work is licensed under a Creative Commons Attribution 4.0 International License. The images or other third party material in this article are included in the article's Creative Commons license, unless indicated otherwise in the credit line; if the material is not included under the Creative Commons license, users will need to obtain permission from the license holder to reproduce the material To view a copy of this license, visit http://creativecommons.org/licenses/by/4.0/

(C) The Author(s) 2016 